\title{
The major thylakoid protein kinases STN7 and STN8 revisited: effects of altered STN8 levels and regulatory specificities of the STN kinases
}

\author{
Tobias Wunder ${ }^{1}$, Wenteng Xu ${ }^{1}$, Qiuping Liu ${ }^{1}$, Gerhard Wanner ${ }^{2}$, Dario Leister ${ }^{1,3 *}$ and Mathias Pribil ${ }^{1,4}$ \\ ${ }_{1}$ Plant Molecular Biology (Botany), Department Biology I, Ludwig-Maximilians-Universität München, Planegg-Martinsried, Germany \\ ${ }^{2}$ Ultrastrukturforschung, Department Biology I, Ludwig-Maximilians-Universität München, Planegg-Martinsried, Germany \\ ${ }^{3}$ PhotoLab Trentino - a Joint Initiative of the University of Trento (Centre for Integrative Biology) and the Edmund Mach Foundation (Research and Innovation \\ Centre), San Michele all'Adige (Trento), Italy \\ ${ }^{4}$ Mass Spectrometry Unit, Department Biology I, Ludwig-Maximilians-Universität München, Planegg-Martinsried, Germany
}

\section{Edited by:}

Mohammad M. Najafpour, Institute for Advanced Studies in Basic

Sciences, Iran

\section{Reviewed by:}

Suleyman I. Allakhverdiev, Russian Academy of Sciences, Russia Harvey J. M. Hou, Alabama State University, USA

\section{*Correspondence:}

Dario Leister, Plant Molecular Biology (Botany), Department Biology I, Ludwig-MaximiliansUniversität München, Großhaderner Str. 2, D-82152 Planegg-Martinsried, Germany

e-mail: leister@Imu.de
Thylakoid phosphorylation is predominantly mediated by the protein kinases STN7 and STN8. While STN7 primarily catalyzes LHCII phosphorylation, which enables LHCII to migrate from photosystem (PS) II to PSI, STN8 mainly phosphorylates PSII core proteins. The reversible phosphorylation of PSII core proteins is thought to regulate the PSII repair cycle and PSII supercomplex stability, and play a role in modulating the folding of thylakoid membranes. Earlier studies clearly demonstrated a considerable substrate overlap between the two STN kinases, raising the possibility of a balanced interdependence between them at either the protein or activity level. Here, we show that such an interdependence of the STN kinases on protein level does not seem to exist as neither knock-out nor overexpression of STN7 or STN8 affects accumulation of the other. STN7 and STN8 are both shown to be integral thylakoid proteins that form part of molecular supercomplexes, but exhibit different spatial distributions and are subject to different modes of regulation. Evidence is presented for the existence of a second redox-sensitive motif in STN7, which seems to be targeted by thioredoxin $f$. Effects of altered STN8 levels on PSII core phosphorylation, supercomplex formation, photosynthetic performance and thylakoid ultrastructure were analyzed in Arabidopsis thaliana using STN8-overexpressing plants (oeSTN8). In general, oeSTN8 plants were less sensitive to intense light and exhibited changes in thylakoid ultrastructure, with grana stacks containing more layers and reduced amounts of PSII supercomplexes. Hence, we conclude that STN8 acts in an amount-dependent manner similar to what was shown for STN7 in previous studies. However, the modes of regulation of the STN kinases appear to differ significantly.

Keywords: chloroplast, protein phosphorylation, PSII supercomplexes, redox, STN kinases, thylakoid ultrastructure

\section{INTRODUCTION}

In Arabidopsis thaliana, the thylakoid kinase STN8 is predominantly responsible for the quantitative phosphorylation of PSII core proteins $(\mathrm{CP} 43, \mathrm{D} 1, \mathrm{D} 2$, and $\mathrm{PsbH})$, particularly under high light conditions (Bonardi et al., 2005; Vainonen et al., 2005; Tikkanen et al., 2010). However, inactivation of STN8 alone does not completely abolish PSII core protein phosphorylation: D1 and D2 phosphorylation falls to about $50-60$ and $35 \%$ of the wildtype level respectively (Vainonen et al., 2005). Only the knock-out of both STN8 and the LHCII kinase STN7 leads to quantitative loss of thylakoid phosphorylation, as monitored by immunodetection (Bonardi et al., 2005; Tikkanen et al., 2008). However, based on MS analyses, Fristedt et al. (2009) were still able to detect residual light-independent $\mathrm{D} 2$ phosphorylation in $\sin 7 \operatorname{stn} 8$ double mutants, corresponding to less than $10 \%$ of the wildtype level. These results reveal a degree of overlap in substrate specificity between STN7 and STN8, although their main targets differ, and suggest that they might act in parallel rather than in series (Bonardi et al., 2005). By combining affinity chromatography with mass spectrometry, Reiland et al. (2011) have identified additional substrates of STN8, including the PGR5-like protein 1A (PGRL1A), which is essential for antimycin A (AA)-sensitive cyclic electron flow (CEF) around photosystem I (DalCorso et al., 2008). The differential phosphorylation of PGRL1A in stn8-1 mutant plants is thought to permit more rapid switching between CEF and linear electron flow (LEF) during dark-light transitions (Reiland et al., 2011). Nevertheless, the function of reversible PSII core phosphorylation, the quantitatively major task of STN8, remains ambiguous.

Initially, the phosphorylated version of photo-damaged D1 was shown to be resistant to proteolysis (Koivuniemi et al., 1995), with the respective PSII complexes being able to move laterally from grana to stroma lamellae for subsequent dephosphorylation, degradation and replacement of damaged D1 (Rintamäki 
et al., 1996). The emerging model suggested that the intensity of PSII core protein phosphorylation was correlated with the increase in damage to PSII reaction centers (D1) as light intensity rises, which would be compatible with an involvement of STN8 in D1 turnover during photoinhibition (Baena-Gonzalez et al., 1999). Making use of the STN kinase mutant collection in A. thaliana, a later study performed by Bonardi et al. (2005) indicated that STN8-mediated phosphorylation of D1 per se is not essential for D1 turnover and PSII repair, challenging the concept that phosphorylation plays a major role in the degradation of D1. Further investigations again provided evidence that lack of STN8 is associated with greater susceptibility to photoinhibition (Nath et al., 2007) and revealed that D1 degradation is delayed in $\operatorname{stn} 8$ and $\sin 7 \operatorname{stn} 8$ mutants exposed to less intense high-light conditions (Tikkanen et al., 2008). Tikkanen et al. (2008) attribute this difference between WT and the $\operatorname{stn} 8$ and $\operatorname{stn} 7 \operatorname{stn} 8$ mutants to disturbances in the disassembly of PSII supercomplexes, leading to less efficient exchange of damaged D1 between grana and stroma lamellae due to changes in its migration behavior. More recent studies by Fristedt et al. (2009) confirmed the observed delay in D1 degradation in stn 8 and $\operatorname{stn} 7 \operatorname{stn} 8$ plants and proposed that the observed increase in the diameter and density of stacked thylakoid membranes (grana) in these lines reduces lateral diffusion of proteins, including photo-damaged D1 and the bulky FtsH complex. The latter is responsible for D1 degradation (Nixon et al., 2005; Adam et al., 2006) and was reported to be spatially separated from PSII in STN8-deficient mutants due to its relocation from the dense grana to the stroma lamellae and grana margins (Fristedt et al., 2009). Therefore, phosphorylation of PSII core proteins is currently assumed to modulate the macroscopic rearrangement of the thylakoid membrane network, as well as the formation of PSII supercomplexes, and to affect lateral movement of proteins within the membrane, thus exerting its effects on D1 turnover indirectly.

In the present study, the effect of increased PSII core phosphorylation in a line overexpressing STN8 (oeSTN8) was analyzed with respect to its impact on PSII supercomplex formation and modulation of thylakoid membrane structure. Furthermore, we analyzed the topology and localization of the STN kinases and show that neither knock-out nor overexpression of STN7 or STN8 affects the accumulation of the other. Finally, we show that STN8 protein levels in wild-type plants do not depend on ambient light conditions, and present evidence for a direct interaction of STN7 with thioredoxins, which is independent of its N-terminal cysteine motif.

\section{MATERIALS AND METHODS PLANT MATERIAL}

The Arabidopsis thaliana L. (A. thaliana) ecotype Columbia0 , used in this study as wild type (WT), was obtained from NASC (Nottingham Arabidopsis Stock Center; accession number N1092). Previously described mutant lines employed in this study were $\operatorname{stn} 7-1, \operatorname{stn} 8-1, \operatorname{stn} 7 \operatorname{stn} 8$ (Bonardi et al., 2005), oeSTN7, STN7C $\longrightarrow$ S:65+70 (Wunder et al., 2013), hcf136 (Meurer et al., 1998), psad1-1 psad2-1 (Ihnatowicz et al., 2004), atpd-1, and petc-1 (Maiwald et al., 2003).

\section{GENERATION OF STN8 OVEREXPRESSOR LINES (OESTN8)}

To generate oeSTN8 lines, the full-length CDS of STN8 was cloned into the plant vector pLeela, which is a derivative of pJawohl3-RNAi (GenBank Accession No. AF404854) containing a GATEWAY cassette introduced into the HpaI site, using the primers Stn8_attB1_ACC_f (GGGGACAAGTT TGTACAAAAAAGCAGGCTCTACCATGGCCTCTCTTCTCTC TC) and Stn8_attB2_Stop_r (GGGGACCACTTTGTACAA GAAAGCTGGGTTTCACTTGCTGAAACTGAGCTT). The STN8-pLeela construct containing a double Cauliflower Mosaic Virus (CMV) 35S promoter was introduced into the stn8-1 mutant background via the floral-dip method (Clough and Bent, 1998). Plants were selected based on their BASTA resistance, segregation analysis was performed, and independent lines carrying a single T-DNA insertion locus were identified. Lines overexpressing the STN8 kinase (oeSTN8) were identified by Western blot analysis employing an STN8-specific antibody.

\section{GROWTH CONDITIONS AND LIGHT TREATMENTS}

If not stated otherwise, plants to be analyzed were grown for 6 weeks under controlled conditions in a growth chamber on an $8 \mathrm{~h} / 16 \mathrm{~h}$ day/night regime providing $100 \mu \mathrm{mol}$ photons $\mathrm{m}^{-2} \mathrm{~s}^{-1}$ during the light phase (standard lighting conditions). For experiments with the mutants hcf136, petc-1, psad1-1 psad2-1, and atpd-1, plants were grown on $1 \times$ MS medium including vitamins (Duchefa ${ }^{\circledR}$ ) at $50 \mu \mathrm{mol}$ photons $\mathrm{m}^{-2} \mathrm{~s}^{-1}$. To study the effects of altered light conditions, plants were adapted to different light conditions specified as follows: $18 \mathrm{~h}$ dark adaptation (D), adaptation to low light at $60-80 \mu \mathrm{mol}$ photons $\mathrm{m}^{-2} \mathrm{~s}^{-1}$ (LL) or high light at $800-1,200 \mu \mathrm{mol}$ photons $\mathrm{m}^{-2} \mathrm{~s}^{-1}(\mathrm{HL})$. The light source used for HL conditions was an Osram Powerstar HQIBT-D/400W lamp. Far-red light (FR) was emitted by LEDs at a wavelength of $740 \mathrm{~nm}$ and an intensity of $3.0 \mu \mathrm{mol}$ photons $\mathrm{m}^{-2} \mathrm{~s}^{-1}$. Adaptation to PSI and PSII light was performed essentially as described previously (Wagner et al., 2008). Briefly, 3-week-old plants were transferred from a climate chamber to either PSI- or PSII-specific light conditions. PSI light $\left(15 \mu \mathrm{mol} \mathrm{m} \mathrm{m}^{-2} \mathrm{~s}^{-1}\right)$ was generated by clamping a medium red foil (Lee Filters, 027 Medium Red, transmittance $50 \%$ at $650 \mathrm{~nm}$ ) over red fluorescent lamps (39 W) from Osram. PSII light $\left(15 \mu \mathrm{mol} \mathrm{m} \mathrm{m}^{-2} \mathrm{~s}^{-1}\right)$ was generated by wrapping an orange foil (Lee Filters, 405 Orange, transmittance $50 \%$ at $560 \mathrm{~nm}$ ) over white fluorescent lamps of the same type.

\section{ISOLATION OF TOTAL PROTEINS}

Total protein extracts were prepared from 6-week-old leaves according to Haldrup et al. (1999). About $0.1 \mathrm{~g}$ of leaf material was homogenized in $200 \mu \mathrm{l}$ solubilization buffer $(100 \mathrm{mM}$ Tris pH 8.0, $50 \mathrm{mM}$ EDTA pH 8.0, 0.25 M NaCl, $1 \mathrm{mM}$ DTT, $0.7 \%$ SDS) and heated to $65^{\circ} \mathrm{C}$ for $10 \mathrm{~min}$. Samples were centrifuged for $10 \mathrm{~min}$ at $10,000 \mathrm{~g}$ to remove insoluble debris and the protein concentration in the supernatant was determined with the amido black assay described by Schaffner and Weissmann (1973). The ubiquitously expressed actin protein was used as a loading control. 


\section{ISOLATION OF THYLAKOID MEMBRANES}

Thylakoids were isolated by a modified procedure based on Bassi et al. (1995). Briefly, leaf material from A. thaliana plants was homogenized in ice-cold isolation buffer $(0.4 \mathrm{M}$ sorbitol, $0.1 \mathrm{M}$ Tricine-KOH pH 7.8, 0.5\% milk powder, $20 \mathrm{mM} \mathrm{NaF}$ ), filtered through two layers of Miracloth (Calbiochem) and centrifuged at $1,500 \mathrm{~g}$ for $10 \mathrm{~min}$ at $4^{\circ} \mathrm{C}$. The membrane pellet was resuspended in ice-cold resuspension buffer $(20 \mathrm{mM}$ HEPES-KOH $\mathrm{pH}$ 7.5, $10 \mathrm{mM}$ EDTA, $20 \mathrm{mM} \mathrm{NaF}$ ) followed by a centrifugation step at $10,000 \mathrm{~g}$ for $10 \mathrm{~min}$ at $4^{\circ} \mathrm{C}$ after $10 \mathrm{~min}$ of incubation on ice. Thylakoids were resuspended in TMK buffer $(10 \mathrm{mM}$ Tris- $\mathrm{HCl}$ $\mathrm{pH}$ 6.8, $10 \mathrm{mM} \mathrm{MgCl}_{2}, 20 \mathrm{mM} \mathrm{KCl,} 20 \mathrm{mM} \mathrm{NaF}$ ). The chlorophyll concentration was determined in aqueous $80 \%$ acetone according to Porra (2002).

\section{IMMUNOBLOT ANALYSIS}

If not stated otherwise, antibodies raised against specific epitopes of STN7 and STN8 were used for Western blot analysis in this study. The peptides CKKVKVGVRGAEEFG of STN8 and LQELREKEPRKKANAQ, located at the C-terminus of STN7, served as immunogens (BioGenes GmbH, Berlin, Germany). Immunoblot analyses with these antibodies, as well as phosphothreonine-specific antibodies (Cell Signaling Technology, Inc., Boston, USA) and polyclonal antibodies raised against actin (Dianova, Germany), RbcL, PsaC, PsaB, PsbO, PsaE, AtpB, Lhcb2, Lhca3 (all Agrisera, Sweden), were performed as previously described (Ihnatowicz et al., 2008).

\section{PAGE ANALYSES}

For Blue-native polyacrylamide gel electrophoresis (BN-PAGE), samples of freshly isolated thylakoids corresponding to $50 \mu \mathrm{g}$ $\mathrm{Chl}$ were resuspended in solubilization buffer $(750 \mathrm{mM} \varepsilon$ aminocaproic acid, $50 \mathrm{mM}$ Bis-Tris $\mathrm{pH} 7.0,5 \mathrm{mM}$ EDTA $\mathrm{pH}$ 7.0, $50 \mathrm{mM} \mathrm{NaCl}$ ) and solubilized for $60 \mathrm{~min}$ with $1.5 \%(\mathrm{w} / \mathrm{v})$ digitonin or for $10 \mathrm{~min}$ with $\mathrm{n}$-dodecyl- $\beta$-D-maltoside $(\beta$-DM) (Sigma) on ice (Pribil et al., 2010). Soluble was then separated from insoluble material by centrifugation $\left(13,100 \mathrm{~g}, 4^{\circ} \mathrm{C}\right)$ for either $70 \mathrm{~min}$ (digitonin) or $10 \mathrm{~min}(\beta-\mathrm{DM})$. After supplementing with $5 \%$ Coomassie brilliant blue G-250 in $750 \mathrm{mM}$ $\varepsilon$-aminocaproic acid, the solubilized material was fractionated by non-denaturing BN-PAGE (4-12\% PA) at $4^{\circ} \mathrm{C}$ as outlined in Heinemeyer et al. (2004). For the second dimension separation, a single lane of the $\mathrm{BN}$ gel was incubated in $2 \times$ Laemmli buffer with $100 \mathrm{mM}$ DTT for $30 \mathrm{~min}$, then placed on top of a SDS gel and subjected to electrophoresis (two-dimensional (2D) BN/SDS-PAGE) (Schottkowski et al., 2009a,b). Standard 12\% SDS-PAGE was performed according to Laemmli (1970) unless indicated otherwise. For non-reducing SDS-PAGE, reducing agents (like DTT) were omitted from the loading dye and samples were not boiled if not otherwise stated.

\section{THYLAKOID FRACTIONATION AFTER STATE 1 AND 2 ADAPTATION}

Plants were acclimated to either state 1 or state 2 light and thylakoid fractionation was performed as previously described (Shapiguzov et al., 2010). Briefly, isolated thylakoids at a concentration of $0.6 \mathrm{mg}$ of chlorophyll $/ \mathrm{mL}$ were solubilized with
$1 \%$ digitonin for $5 \mathrm{~min}$, followed by stepwise centrifugation of supernatants. Pellets collected after centrifugation at $10,000 \mathrm{~g}$, $40,000 \mathrm{~g}$ and $150,000 \mathrm{~g}$ represent enriched grana, grana margins and stroma lamellae fractions, respectively. The samples were analyzed by SDS-PAGE and Western blotting.

\section{SUCROSE-GRADIENT FRACTIONATION OF THYLAKOID PROTEIN COMPLEXES}

To prepare sucrose gradients, $11-\mathrm{mL}$ aliquots of $0.4 \mathrm{M}$ sucrose, $20 \mathrm{mM}$ Tricine- $\mathrm{NaOH}$ ( $\mathrm{pH} 7.5$ ), $0.06 \% \beta$-DM were subjected to three successive freeze-thaw $\left(4^{\circ} \mathrm{C}\right)$ cycles. The gradient was underlaid with a cushion of $1 \mathrm{~mL}$ of $60 \%(\mathrm{w} / \mathrm{v})$ sucrose. Thylakoids, prepared from plants that had been exposed to low light were washed twice with $5 \mathrm{mM}$ EDTA ( $\mathrm{pH} 7.8)$ and diluted to a final chlorophyll concentration of $2 \mathrm{mg} / \mathrm{mL}$. Solubilization with $\beta$ $\mathrm{DM}$ at a final concentration of $1 \%$ was performed on ice for $10 \mathrm{~min}$ and followed by centrifugation $\left(16,000 \mathrm{~g}, 5 \mathrm{~min}, 4^{\circ} \mathrm{C}\right)$. The supernatant was loaded on sucrose gradients and centrifuged at $132,000 \mathrm{~g}$ for $21 \mathrm{~h}$ at $4^{\circ} \mathrm{C}$ in a swing-out rotor (Beckman SW 40). Gradients were divided into 16 fractions, which were electrophoresed on a 15\% SDS-PA gel and analyzed on a Western blot.

\section{CHLOROPLAST ISOLATION AND FRACTIONATION INTO STROMA AND THYLAKOIDS}

Chloroplasts were isolated from A. thaliana leaves as described (Aronsson and Jarvis, 2002). To obtain thylakoid and stroma fractions, chloroplasts were ruptured by adding 10 volumes of lysis buffer (20 mM HEPES-KOH pH 7.5, $10 \mathrm{mM}$ EDTA) and incubating on ice for $30 \mathrm{~min}$. The supernatant and pellet collected after a 30 -min centifugation at $42,000 \mathrm{~g}$ and $4^{\circ} \mathrm{C}$ were used as stroma and thylakoid fractions, respectively.

\section{SALT WASHES OF THYLAKOID MEMBRANES}

Salt washes of thylakoid membranes were basically performed according to Karnauchov et al. (1997). Freshly isolated thylakoids (chlorophyll concentration $0.5 \mathrm{mg} / \mathrm{mL}$ ) were incubated for $30 \mathrm{~min}$ on ice in HS buffer $(0.1 \mathrm{M}$ sucrose, $10 \mathrm{mM}$ HEPES$\mathrm{NaOH} \mathrm{pH}$ 8.0) or HS buffer containing $2 \mathrm{mM} \mathrm{NaCl}, 2 \mathrm{M} \mathrm{NaBr}$, $2 \mathrm{M} \mathrm{NaSCN}, 0.1 \mathrm{M} \mathrm{Na}_{2} \mathrm{CO}_{3}$ or $0.1 \mathrm{M} \mathrm{NaOH}$, respectively. After addition of two volumes of HS buffer, the samples were centrifuged at $13,100 \mathrm{~g}$ for $15 \mathrm{~min}$ at $4^{\circ} \mathrm{C}$. Proteins in the pellet fraction were subsequently solubilized directly in Laemmli buffer, whereas the supernatant was first precipitated in $80 \%$ acetone.

\section{CHLOROPHYLL FLUORESCENCE MEASUREMENTS DURING LIGHT INDUCTION AND PSII INACTIVATION INDUCED BY HIGH LIGHT}

Steady-state photosynthetic parameters were measured under actinic red light of increasing light intensity $(22,37,53,95,216$, $513,825,1,287$, and $1,952 \mu \mathrm{mol}$ photons $\left.\mathrm{m}^{-2} \mathrm{~s}^{-1}\right)$, using the Dual-PAM 100 system (Heinz Walz GmbH, Effeltrich, Germany) in the Dual PAM mode, according to the manufacturer's instructions and with standard settings. Plants were dark-adapted for $10 \mathrm{~min}$ prior to measurements and allowed to adapt for 5 min to each new light level. Five plants of each genotype were analyzed for each measurement using always the sixth 
true leaf of the respective plant. Photoinhibition of photosystem II (PSII) was induced over a period of $10 \mathrm{~h}$ with aid of the Imaging PAM System (Walz) by exposing leaves to blue light alternating every $2 \mathrm{~min}$ between $\mathrm{HL}(1,250 \mu \mathrm{mol}$ photons $\left.\mathrm{m}^{-2} \mathrm{~s}^{-1}\right)$ and LL $\left(10 \mu \mathrm{mol}\right.$ photons $\left.\mathrm{m}^{-2} \mathrm{~s}^{-1}\right)$. Maximum PSII quantum yield $[\mathrm{Fv} / \mathrm{Fm}=(\mathrm{Fm}-\mathrm{Fo}) / \mathrm{Fm}]$ was determined every $60 \mathrm{~min}$ after the LL phase and an additional 5-min dark adaptation.

\section{REDOX TITRATION OF STN7 IN THYLAKOID MEMBRANES}

Thylakoid proteins were isolated and equilibrated on ice for $3 \mathrm{~h}$ with various redox buffers (100 mM MOPS pH 7.0, $330 \mathrm{mM}$ sorbitol) containing DTTred and DTTox in various molar ratios. Reactions were solubilized with $2 \%$ SDS and subsequently separated by non-reducing 15\% SDS-PAGE. After transfer of proteins to PVDF membrane, reduced and oxidized forms of STN7 were detected by immunoblot analysis.

\section{THIOREDOXIN (TRX) AFFINITY PURIFICATION}

Affinity purification was basically performed as described by Motohashi et al. (2001). His-tagged rec $\Delta$ TRX-f $(-m)$ was expressed in E. coli and purified by Ni-NTA resin according to the Qiagen protocol for native protein purification (Expressionist, Qiagen), without eluting resin-bound proteins. An aliquot of isolated thylakoid membranes $\quad=1 \mathrm{mg}$ Chl) was solubilized with $1.5 \%$ digitonin in $50 \mathrm{mM}$ Tris$\mathrm{HCl} \mathrm{pH} 8.0$ for $60 \mathrm{~min}$. After centrifugation at $16,100 \mathrm{~g}$ for $70 \mathrm{~min}$, the supernatant was incubated with the TRXcoupled resin $(\sim 5 \mathrm{mg} \mathrm{TRX} / \mathrm{mL})$ for $60 \mathrm{~min}$ at RT. The column was washed three times with washing buffer $(50 \mathrm{mM}$ Tris- $\mathrm{HCl} \mathrm{pH}$ 8.0, $200 \mathrm{mM} \mathrm{NaCl}, 0.2 \%$ digitonin) and proteins retained by thioredoxin were eluted by adding $10 \mathrm{mM}$ DTT. Samples were analyzed on a Western blot, using STN7-specific antibodies.

\section{MOBILITY SHIFT ASSAY OF TRX}

For the TRX mobility shift assay, $10 \mu \mathrm{g}$ of thylakoids were solubilized with $0.2 \%$ deoxycholic acid (DOC) and incubated with $25 \mu \mathrm{g}$ of recombinant TRX-f (rec $\Delta$ TRX-f) for $30 \mathrm{~min}$ in $100 \mathrm{mM}$ MOPS (pH 7.0) and $330 \mathrm{mM}$ sorbitol at RT. Untreated thylakoids (in $0.2 \%$ DOC buffer) served as a control. Subsequently, protein mixtures were subjected to non-reducing SDS-PAGE and immunoblotting.

\section{ANALYSIS OF THYLAKOID MEMBRANE ULTRASTRUCTURE BY TRANSMISSION ELECTRON MICROSCOPY (TEM)}

Plants were grown for 4 weeks in the climate chamber on a $12 \mathrm{~h} / 12 \mathrm{~h}$ day/night regime. $1.5 \mathrm{~h}$ after the onset of the light phase the sixth rosette leaf was cut off and fixed for $1 \mathrm{~h}$ with $2.5 \%$ glutaraldehyde in fixation buffer $(75 \mathrm{mM}$ cacodylic acid, $2 \mathrm{mM}$ $\mathrm{MgCl}_{2} \mathrm{pH}$ 7.0). The material was washed with buffer, incubated for $2 \mathrm{~h}$ with $1 \%$ osmium tetroxide in fixation buffer, washed again with fixation buffer and finally with distilled water. Samples were dehydrated by sequential incubation in increasing acetone concentrations, embedded in resin and sectioned with a microtome after complete polymerisation. Micrographs of the sections were taken with an EM-912 electron microscope (Zeiss) equipped with an integrated OMEGA energy filter operated in the zero-loss mode.

\section{RESULTS}

\section{STN7 AND STN8 ARE NOT LINKED BY A FEEDBACK LOOP}

Previous studies had demonstrated a significant substrate overlap between the kinases STN7 and STN8 (Bonardi et al., 2005). To investigate whether alterations in STN7 levels affect STN8 accumulation and vice versa, $A$. thaliana mutant plants either lacking (stn7-1 and $\operatorname{stn} 8-1)$ or overexpressing (oeSTN7 and oeSTN8) one of the kinases were analyzed. To this end, STN8 overexpressor lines (oeSTN8) were generated that express STN8 under the control of the $35 \mathrm{~S}$ promoter in the $\operatorname{stn} 8-1$ mutant background. This resulted in an increase of 16-fold or more in STN8 protein levels relative to the WT (Figure 1A). The analysis of STN7 and STN8 proteins in oeSTN8 plants and the previously described mutant lines stn7-1, stn8-1 and oeSTN7 (Bonardi et al., 2005; Wunder et al., 2013) using epitope-specific STN antibodies (Figures 1B-D) revealed no significant alterations in the level of the genetically unperturbed kinase. Thus, fluctuations in the availability of one STN kinase do not lead to compensatory changes in the concentration of the other one.

\section{THE STN KINASES ARE THYLAKOID INTEGRAL MEMBRANE PROTEINS WITH DISTINCT SPATIAL DISTRIBUTIONS THAT FORM PART OF LARGE PROTEIN COMPLEXES}

Previous evidence for the localization of the STN8 kinase was based on in-vitro import studies with pea chloroplasts (Bonardi et al., 2005). However, localization studies of STN8 with specific antibodies have not been performed. For this purpose, WT A. thaliana chloroplasts were fractionated into soluble and membrane components and subjected to immunoblot analysis using antibodies raised against a defined STN8 epitope. STN8 was detected exclusively in the membrane fraction, ruling out the existence of a soluble variant of the protein (Figure 2A). The purity of the respective fractions was confirmed by the detection of stroma (RbcL) and thylakoid membrane (Lhcb2) marker proteins. To clarify whether the hydrophobic moieties of the STN kinases truly represent predicted transmembrane domains (TMDs) (Vainonen et al., 2005) or mediate extrinsic attachment to the thylakoid membrane only, WT thylakoids were treated with alkaline buffers or chaotropic agents. Both STN7 and STN8 behaved like the Rieske protein (PetC) (Figure 2B), which has been shown to possess a single hydrophobic domain and to associate with the thylakoid membrane predominantly via electrostatic interactions (Karnauchov et al., 1997). Therefore, we conclude that both STN kinases constitute integral membrane proteins as previously suggested (Vainonen et al., 2005; Lemeille et al., 2009).

To monitor possible changes in the sub-thylakoid localization of the STN kinases under either STN7/8-activating or -inhibiting light conditions, thylakoids from WT plants were isolated after exposure to PSI- or PSII-specific light, and fractionated via differential centrifugation after solubilization with $1.5 \%$ digitonin. While STN8 was enriched in the grana membranes (10K fraction), STN7 was predominantly located in the stroma lamellae (150K fraction) (Figure 3A). The distributions of STN7 and 


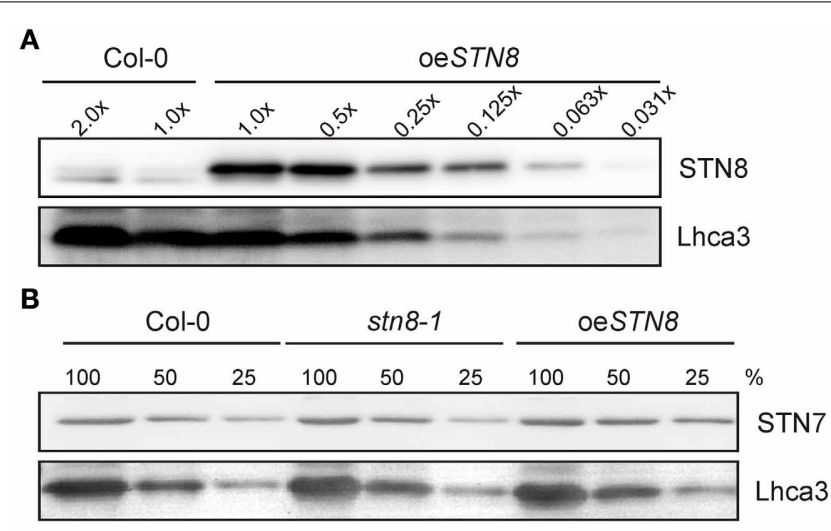

C

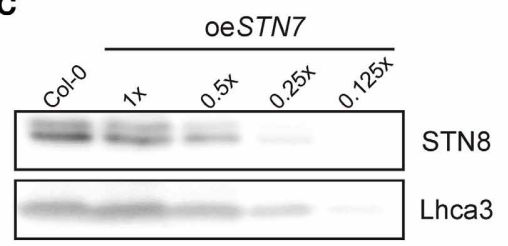

D

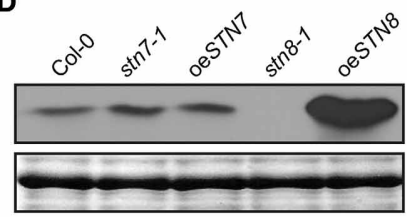

STN8 CBB

FIGURE 1 | Non-interdependence of STN kinase accumulation. (A) WT (Col-0) thylakoid proteins corresponding to $10 \mu \mathrm{g}(2.0 x)$ and $5 \mu \mathrm{g}(1.0 x)$ of $\mathrm{Chl}$ and serial dilutions of oeSTN8 thylakoids were loaded to quantify the levels of STN8 protein in oeSTN8 plants. Proteins were subjected to SDS-PAGE and Western blot analysis using STN8- and Lhca3-specific antibodies. (B) STN7 accumulation in thylakoids of WT (Col-0), oeSTN8 and stn8-1 mutant plants. Thylakoid proteins, corresponding to $8 \mu \mathrm{g}(100 \%), 4 \mu \mathrm{g}(50 \%)$, and $2 \mu \mathrm{g}$ (25\%) of Chl, from each genotype were loaded on a SDS gel and probed using specific antibodies against STN7 and Lhca3 (as loading control). (C)
Accumulation of STN8 in thylakoids of WT (Col-0) and oeSTN7. Thylakoids of WT corresponding to $5 \mu \mathrm{g}$ of $\mathrm{Chl}$ and serial dilutions of oeSTN7 thylakoid proteins were separated by SDS-PAGE, and immunoblots were probed with antibodies specific for STN8 and Lhca3 (as loading control). (D) Thylakoids of WT (Col-0), stn8-1, stn7-1, oeSTN8, and oeSTN7 plants corresponding to $5 \mu \mathrm{g}$ of $\mathrm{Chl}$ were analyzed by Western blotting with antibodies raised against an STN8-specific (left panel) or an STN7-specific (right panel) peptide fragment. Replicate SDS-gels were stained with Coomassie brilliant blue (CBB) and the section with the LHCII signal is shown as loading control.
STN8 between the different membrane fractions were not affected by exposure to PSI- or PSII-favoring light conditions. These data indicate that the major fraction of STN8 resides within the grana stacks close to its assumed primary substrates - the subunits of PSII - irrespective of light conditions. The major proportion of STN7 was localized to the stroma lamellae, with only a minor fraction being found in the grana margins or grana stacks. The distribution of STN7 thus coincides with that of PetC, a subunit of the Cyt $b_{6} f$ complex known to interact physically with Stt7, the Chlamydomonas homolog of STN7 (Lemeille et al., 2009) (Figure 3A).

To further refine the localization of the STN kinases within the thylakoid membrane, their association with known thylakoid protein complexes was investigated. To this end, WT thylakoids were solubilized with $1.6 \% \beta$-DM, separated on BN-PA gels and subsequently resolved via 2D-PAGE. Both STN7- and STN8-specific antibodies gave rise to signals covering the entire molecular weight range, from large supercomplexes down to the free protein fraction (Figure 3B), suggesting that they associate at least weakly with some of the major photosynthetic complexes.
Thylakoids were also fractionated by ultracentrifugation on a linear sucrose gradient after $\beta$-DM solubilization. Again, STN7 and STN8 were both identified in fractions containing high-molecular-weight (HMW) complexes (Figure S1A). These results agree with previous findings showing that $\mathrm{Stt} 7$ associates with a HMW complex (Lemeille et al., 2009), indicating that neither of the STN kinases normally occurs as a monomeric polypeptide, but remains in close association with HMW complexes.

The association of STN8 with HMW complexes was also probed by analyzing different mutant plants devoid of one of the major photosynthetic complexes, following analogous studies previously performed for STN7 (Wunder et al., 2013). Here, absence of PSII (in $h c f 136$ ) also prevented STN8 accumulation, which is in agreement with their co-localization to grana lamellae (Figure S1B). Moreover, a strong decrease in STN8 levels was observed in plants lacking PSI ( $p s a d 1-1$ psad2-1), which is probably due to a significant concomitant reduction in PSII amounts rather than to the loss of PSI per se. Thus, the presence of PSII, the major constituent of thylakoid protein supercomplexes, seems to be a prerequisite for STN8 accumulation. 

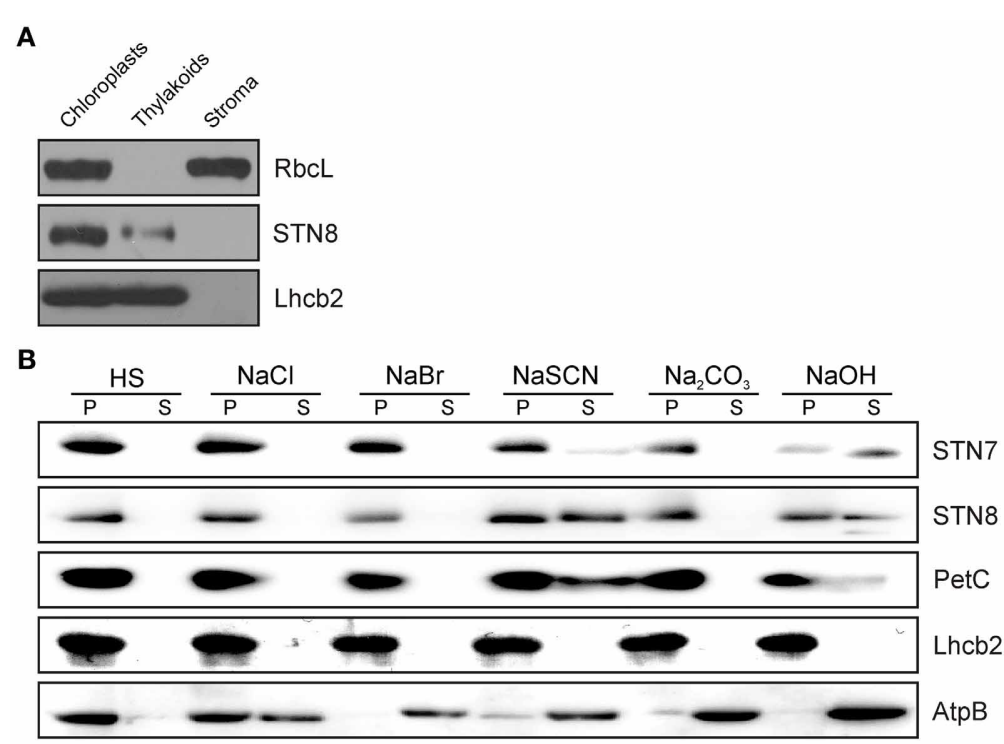

FIGURE 2 | Sub-organellar localization of STN8 and membrane association of the STN kinases. (A) Arabidopsis WT chloroplasts were fractionated into soluble and membrane components and subsequently separated by SDS-PAGE. After Western transfer, STN8, RbcL (stroma marker) and Lhcb2 (thylakoid marker) were detected using specific antibodies. (B) Extraction of membrane-associated proteins with alkaline buffers or chaotropic salt solutions. Thylakoid membranes from WT plants were resuspended at $0.5 \mathrm{mg}$ chlorophyll/mL in HS buffer containing either $2 \mathrm{M} \mathrm{NaCl}, 0.1 \mathrm{M} \mathrm{NaBr}, 2 \mathrm{M} \mathrm{NaSCN}, 0.1 \mathrm{M} \mathrm{Na}_{2} \mathrm{CO}_{3}$, $0.1 \mathrm{M} \mathrm{NaOH}$, or no additive. After incubation on ice, samples were fractionated into soluble $(S)$ and membrane-associated proteins $(P)$ and immunolabeled with antibodies specific for STN7, STN8, AtpB (a representative peripheral membrane protein), PetC (a membrane protein with a single hydrophobic domain predominantly anchored by electrostatic interactions), or Lhcb2 (an integral membrane protein with three transmembrane helices).

\section{REDOX SENSITIVITY OF STN7}

As recently demonstrated, STN7 activity is redox sensitive, and depends on an N-terminal cysteine motif (Wunder et al., 2013). To analyze possible redox-based effects on the tertiary structure of STN7, thylakoids of WT and STN7 $\mathrm{C} \longrightarrow \mathrm{S}: 65+70$ mutant plants devoid of the $\mathrm{N}$-terminal redox-sensitive cysteine motif (Wunder et al., 2013) were incubated in a series of buffers containing reduced and oxidized DTT in different molar ratios, and then subjected to non-reducing SDS-PAGE. Interestingly, both wild-type STN7 and STN7 $\mathrm{C} \longrightarrow \mathrm{S}: 65+70$ monomers showed a clear size-shift in response to the redox treatment (Figure 4A), suggesting the occurrence of a redox-dependent conformational change in STN7 or the release of a possible redox-sensitive co-factor. This implies that the N-terminal CxxxxC motif is not the only redoxsensitive site in the STN7 protein. Under progressively more reducing conditions the amounts of STN7 detected increased significantly. This may indicate that under oxidizing conditions STN7 associates with various HMW aggregates/complexes, such that less STN7 enters the gel in the absence of reduced DTT. Also the STN7 epitope that is recognized by the peptide-specific antibody might be more accessible under reducing conditions.

STN7 activity, especially its inactivation under HL, is thought to be regulated via stromal thioredoxins such as TRX-f and -m (Rintamäki et al., 2000; Lemeille and Rochaix, 2010). So to search for a direct interaction between STN7 and TRX-f and/ or - $m$, recombinant TRX-f and TRX-m variants containing a single Cysto-Ser exchange in their catalytic CGPC motifs (rec $\Delta T R X s$ ) were generated. Due to this single cysteine mutation in the conserved
TRX motif, stable covalent bonds instead of a transient interaction are formed during the disulfide bridge interchange reaction with the substrate. In this way, potential targets of TRXs are trapped as stable dimeric intermediates. To confirm such covalent binding of STN7 to rec $\triangle T R X s$, WT thylakoids were solubilized with $1.5 \%(\mathrm{w} / \mathrm{v})$ digitonin and incubated with either rec $\Delta \mathrm{TRX}$ $\mathrm{f}$ or rec $\Delta$ TRX-m immobilized on Ni-NTA resin. After several washing steps proteins interacting with rec $\Delta T R X-f$ and $-m$ were eluted with a DTT-containing buffer, and the presence of STN7 was determined via Western analysis. Although slightly shifted in size, STN7 could indeed be detected in the fraction eluted from the rec $\triangle T R X-f$ resin, whereas no STN7 was detectable in the same fraction from the rec $\Delta \mathrm{TRX}-\mathrm{m}$ column (Figure 4B). In addition, the notion that STN7 can covalently bind to rec $\Delta$ TRX-f was supported by a significant decrease in amounts of STN7 in the flow-through in comparison to the input fraction (Figure 4B).

To further assess the possible interaction of STN7 with TRX-f, a TRX mobility-shift assay was performed. To this end, rec $\triangle T R X-f$ was incubated with solubilized thylakoids from $\mathrm{WT}, \operatorname{stn} 7-1$ and $\mathrm{STN}_{\mathrm{C}} \longrightarrow \mathrm{S}: 65+70$ plants (Figure 4C). Covalent binding of rec $\triangle T R X-f$ to STN7 is expected to reduce the electrophoretic mobility of the STN7 signal due to the increased molecular weight of the resulting complex, but no STN7-TRX-f linkage product was detectable by Western analysis. However, the STN7 monomer disappeared almost completely upon addition of TRX-f (Figure 4C), suggesting a direct interaction between the two. Whether TRX-f treatment led to precipitation of STN7 or to formation of cross-linked, HMW aggregates in which 


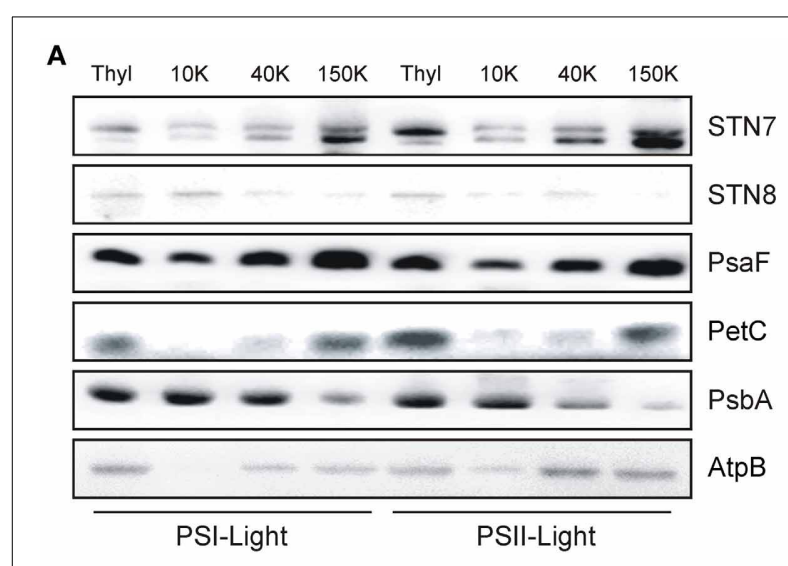

B

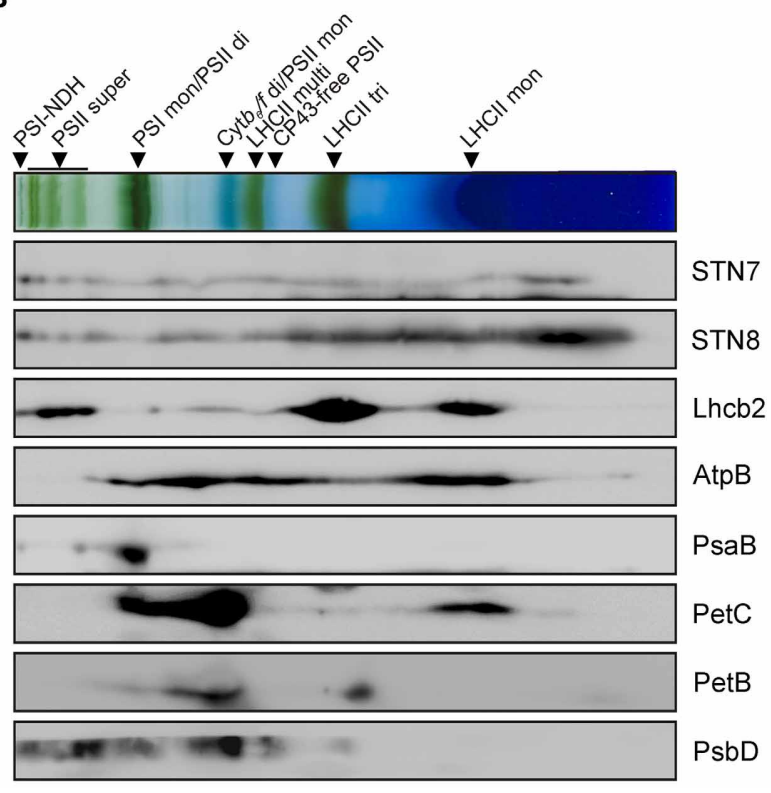

FIGURE 3 | Localization of the STN kinases within the thylakoid membrane. (A) Analysis of STN7 and STN8 localization by thylakoid membrane sub-fractionation. Thylakoids of PSII- or PSI-light adapted WT plants were fractionated into grana lamellae $(10 \mathrm{~K})$, grana margins $(40 \mathrm{~K})$ and stroma lamellae $(150 \mathrm{~K})$ by differential ultracentrifugation after digitonin solubilization. Proteins were separated by SDS-PAGE and analyzed by immunolabeling using antibodies against STN7, STN8, PsaF, PetC, PsbA, and AtpB. (B) Analysis of STN7 and STN8 distribution by 2D BN/SDS-PAGE. Thylakoid membranes of WT equivalent to $50 \mu \mathrm{g}$ of $\mathrm{Chl}$ were solubilized with $\beta$-DM, subjected to $2 \mathrm{D}$ BN/SDS-PAGE and analyzed by Western blotting. STN7, STN8 and various subunits of the major photosynthetic complexes (AtpB, Lhcb2, PsaB, PetC, PetB, and PsbD) were detected with specific antibodies. Protein-Chl complexes are indicated by black arrowheads. The bands detected were identified as specific protein complexes in accordance with previously published profiles (Armbruster et al., 2013): PSI-NDH supercomplex (PSI-NDH), PSII supercomplexes (PSII super), PSI monomers and PSII dimers (PSI mon and PSII di), PSII monomers and dimeric Cyt $b_{6} / f$ (PSII mon and $C y t b_{6} / f$ di), PSII monomers w/o CP43 (CP43-free PSII), multimeric (LHCII multi), trimeric (LHCII tri), and monomeric (LHCII mon) LHCII.

the STN7 epitope is inaccessible to the antibody could not be definitively clarified. Interestingly, the $S T N 7 \mathrm{C} \longrightarrow \mathrm{S}: 65+70$ variant behaved like wild-type STN7 (Figure 4C), which supports the inference from the redox titration assay (Figure 4A) regarding the existence of a second redox-sensitive site within STN7.

\section{STN8 PROTEIN LEVELS DO NOT RESPOND TO CHANGES IN LIGHT CONDITIONS}

STN8 activity was previously shown to be regulated by changes in light quality and quantity (Bonardi et al., 2005; Tikkanen et al., 2010). In particular, FR and HL treatments led to a decrease and increase in PSII core protein phosphorylation respectively (Tikkanen et al., 2008, 2010). To investigate whether these changes are due to alterations in STN8 level or activity, WT plants were exposed for $2 \mathrm{~h}$ to LL after $18 \mathrm{~h}$ of darkness and subsequently transferred for $4 \mathrm{~h}$ to FR or HL $\left(800 \mu\right.$ mol photons $\left.\mathrm{m}^{-2} \mathrm{~s}^{-1}\right)$, after which the amounts of STN8 present were determined. In contrast to STN7 (Wunder et al., 2013), STN8 levels did not change markedly upon these light-shift treatments, and showed no significant variations even after exposure to LL for up to $9 \mathrm{~h}$ (Figure 5). This stable expression profile suggests that STN8 is regulated at the level of specific activity rather than amount.

\section{OVEREXPRESSION OF STN8 INCREASES PSII PROTEIN PHOSPHORYLATION AND DISASSEMBLY OF PSII SUPERCOMPLEXES UNDER HL CONDITIONS}

To determine the effects of increased STN8 accumulation on thylakoid protein phosphorylation, WT, stn8-1, $\sin 7 \operatorname{stn} 8$ and oeSTN8 plants were dark-adapted for $18 \mathrm{~h}$, then exposed for $2 \mathrm{~h}$ to $\mathrm{LL}\left(80 \mu \mathrm{mol}\right.$ photons $\left.\mathrm{m}^{-2} \mathrm{~s}^{-1}\right)$ followed by either $4 \mathrm{~h}$ of $\mathrm{HL}$ $\left(1,000 \mu \mathrm{mol}\right.$ photons $\left.\mathrm{m}^{-2} \mathrm{~s}^{-1}\right)$ or $2 \mathrm{~h}$ of FR. The phosphorylation status of isolated thylakoids was then monitored by Western blot using phosphothreonine-specific antibodies (Figure 6A). In general, oeSTN8 plants exhibited a significant increase in PSII core protein phosphorylation under all investigated light conditions. While PSII phosphorylation in WT plants decreased in the dark compared to LL conditions, PSII phosphorylation levels in oeSTN8 lines remained high under both conditions. After FR treatment, residual PSII phosphorylation was detectable only in oeSTN8. The strongest increase in PSII core protein phosphorylation in oeSTN8 relative to WT was observed under $\mathrm{HL}$, which is known to induce STN8 activity (Bonardi et al., 2005). Interestingly, pLHCII levels differed little between oeSTN8 and WT, suggesting that the excess STN8 present in oeSTN8 plants does not significantly affect the phosphorylation status of STN7-specific substrates under the light conditions examined (Figure 6A).

Under HL conditions, thylakoid phosphorylation - in particular phosphorylation of the PSII core proteins - has been shown to promote the disassembly of PSII supercomplexes (Tikkanen et al., 2008). To examine effects of increased PSII protein phosphorylation on PSII supercomplexes, BN-PAGE analyses were performed on thylakoids after exposure of oeSTN8 plants to $18 \mathrm{~h}$ $\mathrm{D}$ and $2 \mathrm{~h} \mathrm{LL}\left(80 \mu \mathrm{mol}\right.$ photons $\left.\mathrm{m}^{-2} \mathrm{~s}^{-1}\right)$ or $2 \mathrm{~h} \mathrm{HL}(1,200 \mu \mathrm{mol}$ photons $\left.\mathrm{m}^{-2} \mathrm{~s}^{-1}\right)$. Solubilization of thylakoid samples from HLtreated plants with $1.5 \%$ digitonin prior to BN-PAGE revealed no significant differences between WT and $\operatorname{stn} 8$ - 1 , while a slight decrease in the amount of PSII supercomplexes was noted in oeSTN8 (Figure 6B, left panel). Similar, but more pronounced, effects on PSII supercomplex accumulation were observed when 


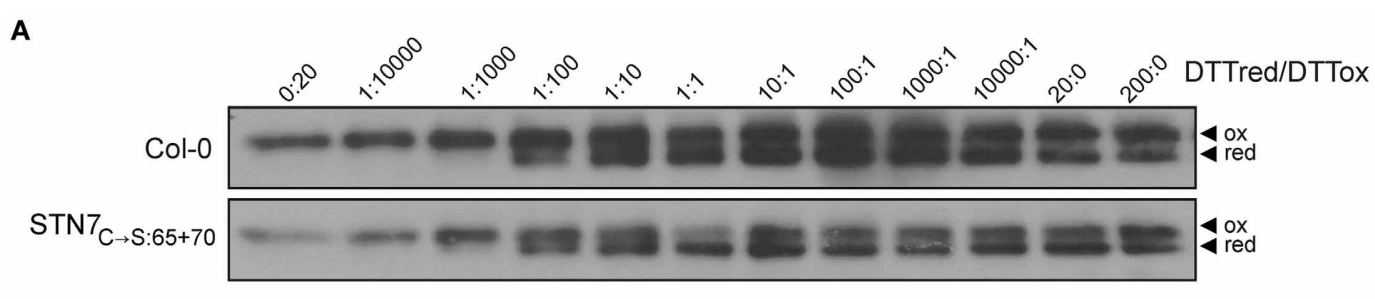

B

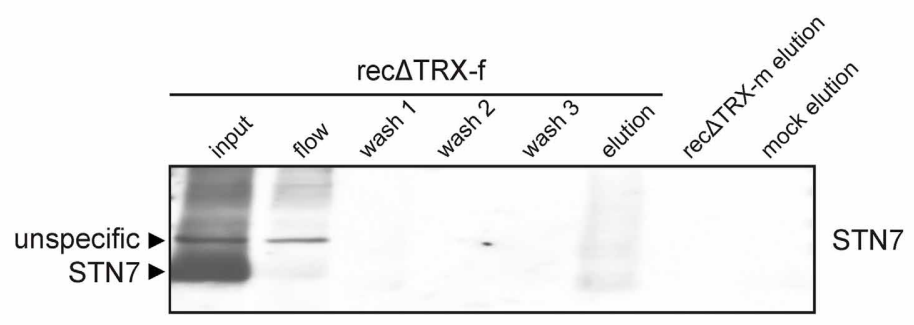

C

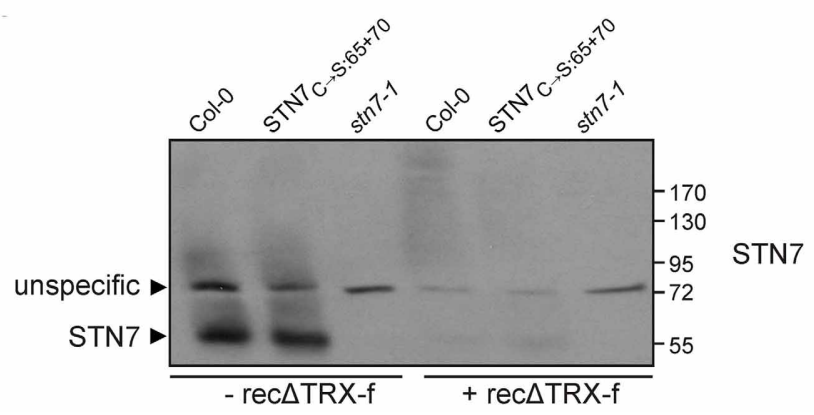

FIGURE 4 | Redox sensitivity of STN7. (A) Redox titration of STN7 variants. Thylakoids of WT $(\mathrm{Col}-0)$ and $S T N 7 \mathrm{C} \longrightarrow \mathrm{S}: 65+70$ were equilibrated at various redox potentials using the DTTred/DTTox redox couple. After separation by non-reducing SDS-PAGE, STN7 was detected by immunoblotting. DTTred/DTTox ratios are indicated above each lane. Oxidized (ox) and reduced (red) forms of the STN7 monomer are indicated by black arrowheads. (B) STN7-TRX-f interaction assayed by thioredoxin affinity chromatography. His-tagged recombinant thioredoxins $f$ and $m$, with one cysteine replaced by serine (rec $\Delta T R X-f,-m)$, were bound to Ni-NTA and incubated with thylakoids solubilized with $1.5 \%$ digitonin corresponding to $2 \mathrm{mg}$ of Chl. After several washes, the eluates from rec $\Delta T R X-f$ and $-m$ resins were subjected to Western blot analysis using STN7-specific antibodies. Solubilized WT (Col-0) thylakoids (input; corresponding to $10 \mu \mathrm{g}$ of Chl), the flow through off the rec $\Delta T R X-f$ resin (flow; equivalent to $10 \mu \mathrm{g} C h l$ ), washes 1-3 of the rec $\Delta T R X-f$ resin and the eluate from a similarly treated Ni-NTA resin not coupled to rec $\Delta T R X$ (mock elution) were loaded as controls. (C) TRX-f mobility shift assay. Thylakoid membranes (10 $\mu \mathrm{g}$ Chl) from WT $(\mathrm{Col}-0), \mathrm{STN}_{\mathrm{C}} \longrightarrow \mathrm{S}: 65+70$ and stn7-1 mutants were solubilized with $0.2 \%$ DOC and incubated with either $25 \mu \mathrm{g}$ of rec $\Delta$ TRX-f or without additives. Proteins were separated by non-reducing SDS-PAGE and Western analysis was performed using STN7-specific antibodies.

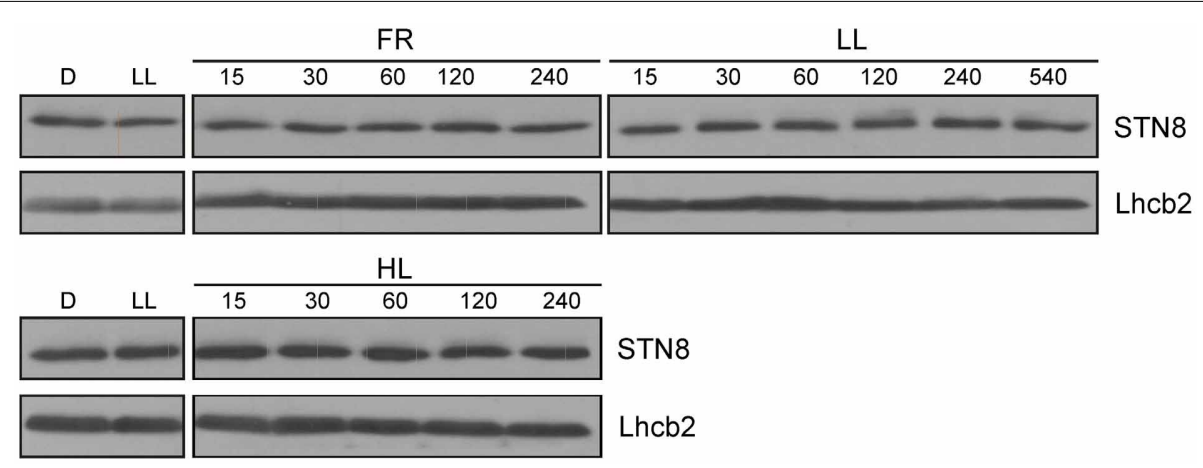

FIGURE 5 | STN8 protein accumulation upon exposure to D, LL, FR, and

HL. WT plants were dark-adapted for $18 \mathrm{~h}$, transferred to low light (LL; $80 \mu \mathrm{mol}$ photons $\mathrm{m}^{-2} \mathrm{~s}^{-1}$ ) for $2 \mathrm{~h}$ and then to $\mathrm{FR}$ or $\mathrm{HL}(\mathrm{HL} ; 800 \mu \mathrm{mol}$ photons $\mathrm{m}^{-2} \mathrm{~s}^{-1}$ ) for an additional $4 \mathrm{~h}$. Leaf material was collected after each treatment and thylakoids were fractionated by SDS-PAGE and analyzed using antibodies specific for STN8 and Lhcb2 (loading control). 
A

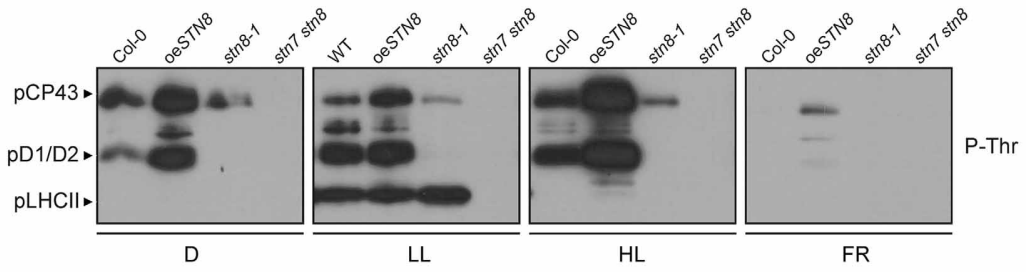

B

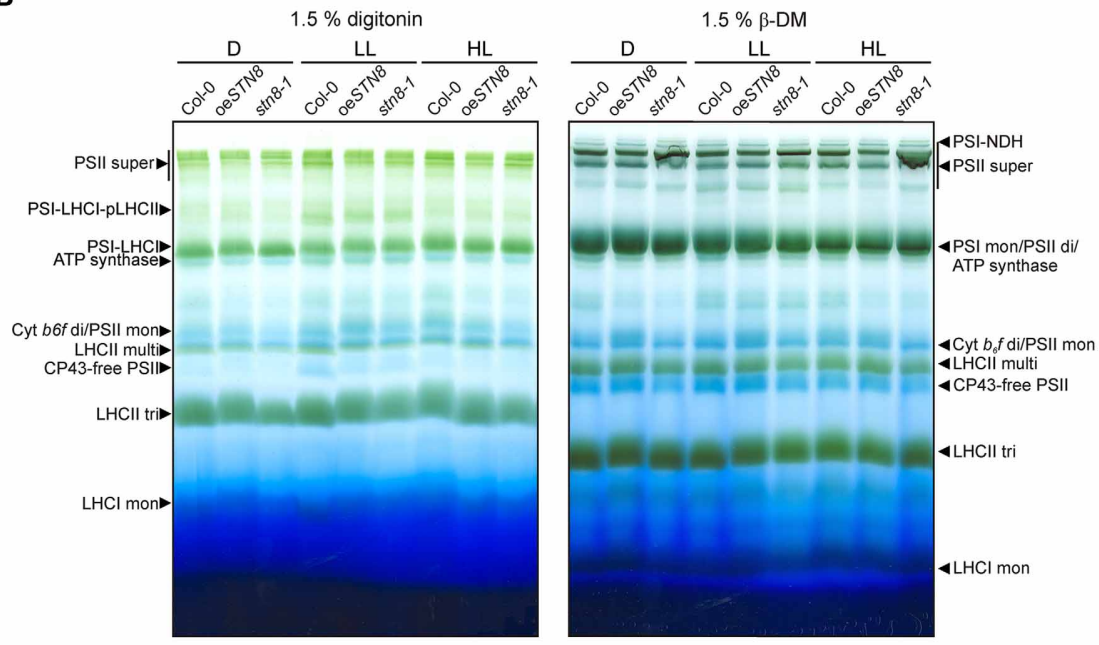

C

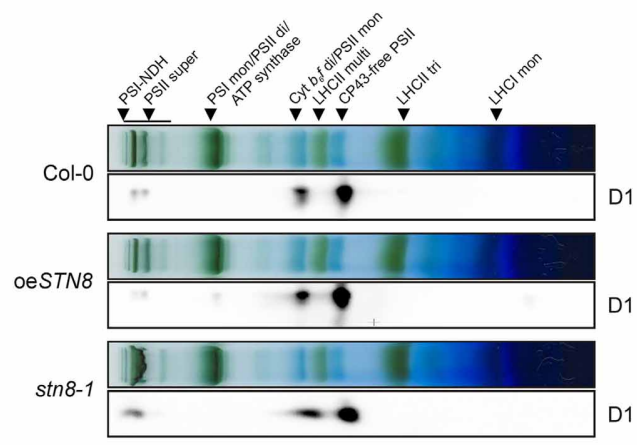

FIGURE 6 | Effects of altered STN8 levels on thylakoid phosphorylation and PSII supercomplex formation. (A) Thylakoid protein phosphorylation in WT (Col-0), oeSTN8, stn8-1, and $\operatorname{stn} 7 \operatorname{stn} 8$ plants dark-adapted for $18 \mathrm{~h}$ (D), subsequently transferred to LL ( $80 \mu \mathrm{mol}$ photons $\mathrm{m}^{-2} \mathrm{~s}^{-1}$ ) for $2 \mathrm{~h}$ and for additional $2 \mathrm{~h}$ to $\mathrm{FR}$ or $4 \mathrm{~h}$ to $\mathrm{HL}\left(1,000 \mu \mathrm{mol}\right.$ photons $\left.\mathrm{m}^{-2} \mathrm{~s}^{-1}\right)$. After these light treatments, isolated thylakoids were analyzed by immunodetected using phosphothreonine (pThr) -specific antibodies (Cell Signaling). The positions of phosphorylated LHCII (pLHCII), CP43 (pCP43) and D1/2 (pD1/2) are indicated by black arrowheads. (B) Patterns of protein complexes solubilized from WT (Col-0), oeSTN8 and stn8-1 thylakoids and separated on BN-PA gels. Thylakoid membranes of WT, oeSTN8 and stn8-1 plants exposed to either $\mathrm{LL}\left(80 \mu \mathrm{mol}\right.$ photons $\left.\mathrm{m}^{-2} \mathrm{~s}^{-1}\right)$ or $\mathrm{HL}\left(1,200 \mu \mathrm{mol}\right.$ photons $\left.\mathrm{m}^{-2} \mathrm{~s}^{-1}\right)$ for $2 \mathrm{~h}$, or kept in the dark for $18 \mathrm{~h}$, were solubilized with $1.5 \%$ digitonin (left panel) or $1.5 \% \beta$-DM (right panel) and separated by BN-PAGE. Protein-Chl complexes are indicated by black arrowheads. The bands detected were identified as specific protein complexes in accordance with previously published profiles (Armbruster et al., 2013): PSI-LHCl-pLHCII supercomplex (PSI-LHCl-pLHCII), PSI-LHCl supercomplex (PSI-LHCI), PSI-NDH supercomplex (PSI-NDH), PSII supercomplexes (PSII super), PSI monomers and PSII dimers (PSI mon and PSII di), PSII monomers and dimeric Cyt $b_{6} f$ (PSII mon and Cyt $b_{6} f$ di), PSII monomers w/o CP43 (CP43-free PSII), multimeric (LHCII multi), trimeric (LHCII tri) and monomeric (LHCII mon) LHCII. (C) BN-PAGE lanes bearing HL-treated samples corresponding to those shown in the right panel of (B) were subjected to SDS-PAGE in the second dimension and probed with D1-specific antibodies. thylakoids were solubilized with $1.5 \% \beta$-DM. Here, WT plants exposed to HL once again contained more PSII supercomplexes than oeSTN8 and fewer than stn8-1 (Figure 6B, right panel), which is in accordance with earlier reports (Tikkanen et al., 2008).

2D-PAGE analysis was performed on HL-treated samples to assess the distribution of the PSII core proteins between PSII supercomplexes, dimers and monomers. Greater accumulation of D1 in PSII supercomplexes was observed in $\operatorname{stn} 8-1$ compared to WT, whereas the opposite was the case for oeSTN8 (Figure 6C). In oeSTN8 a clear shift in the ratio of PSII supercomplexes to PSII dimers and monomers in favor of the latter two was observed in comparison to the situation in WT and the stn8-1 mutant. 
This suggests that disassembly of PSII supercomplexes is indeed enhanced by an increase in PSII core protein phosphorylation, as previously described (Tikkanen et al., 2008).

\section{INCREASED STN8 LEVELS REDUCE SUSCEPTIBILITY TO PSII PHOTOINHIBITION AND LEAD TO A MODERATE RISE IN THE OXIDATION STATE OF THE PO POOL}

In previous studies, a decrease in the turnover of damaged D1 was observed in leaves of $\operatorname{stn} 7 \operatorname{stn} 8$ and $\operatorname{stn} 8-1$ plants exposed to high light. This was attributed to the lack of PSII core protein phosphorylation, which is a prerequisite for the disassembly of PSII supercomplexes and the migration of damaged PSII complexes from grana to stroma lamellae (Tikkanen et al., 2008; Fristedt et al., 2009). Accordingly, a reduction in PSII phosphorylation is expected to increase sensitivity to photoinhibition during HL treatment, which translates into lower Fv/Fm values relative to WT. To address the question whether increased PSII phosphorylation levels affect susceptibility to photoinhibition, WT, $\sin 8-1$ and oeSTN8 plants were exposed to fluctuating light intensities by switching from LL $\left(10 \mu \mathrm{mol}\right.$ photons $\left.\mathrm{m}^{-2} \mathrm{~s}^{-1}\right)$ to HL $\left(1,250 \mu \mathrm{mol}\right.$ photons $\left.\mathrm{m}^{-2} \mathrm{~s}^{-1}\right)$ and back every $3 \mathrm{~min}$. While stn8-1 and WT plants did not differ significantly in their Fv/Fm values, as previously shown (Bonardi et al., 2005), Fv/Fm values in oeSTN8 plants were slightly higher than in WT (Figure 7A). This can be explained if one assumes that light fluctuations enhance the positive effect of elevated STN8 levels at the onset of HL by allowing a more rapid response to sudden light stress. Overall, enhanced disassembly of PSII supercomplexes under HL conditions, as in the case of oeSTN8 (Figure 6), seems to have only a moderate effect on PSII repair.

To explore general effects of altered PSII core phosphorylation on photosynthesis, $\mathrm{Chl}$ a fluorescence and absorption parameters were recorded for WT, oeSTN8, stn8-1 and $\sin 7 \operatorname{stn} 8$ plants during dark-light transitions (Figures 7B,C). When dark-adapted plants were exposed to LL ( $22 \mu \mathrm{mol}$ photons $\mathrm{m}^{-2} \mathrm{~s}^{-1}$ ) for $6 \mathrm{~min}$, no significant differences in the effective quantum yield of PSII $\left(\Phi_{\mathrm{II}}\right)$ were detected between WT and stn8-1, while in oeSTN8 plants the $\Phi_{\mathrm{II}}$ was initially higher and gradually converged to WT levels during the course of the measurement (Figure 7B). 1-qL, a measure of the excitation pressure of PSII, was somewhat lower in oeSTN8 compared to WT and $\operatorname{stn} 8$-1, which suggests a slightly more oxidized PQ pool (Figure 7C). As expected, the $\sin 7 \sin 8$ mutant showed higher 1-qL and lower $\Phi_{\text {II }}$ values than the WT. Due to the slightly increased resistance of oeSTN8 plants to photoinhibition (Figure 7A), a degree of alteration in photosynthetic performance could be expected even under short-term exposure to high light. However, upon stepwise increase in light intensity (in 5-min steps), $\Phi_{\mathrm{II}}$ and 1-qL measurements revealed no significant differences between oeSTN8 and WT plants (Figures 7D-F). Non-photochemical quenching (NPQ) alone showed a slight increase in oeSTN8 under strong light intensities, as in the case of $\sin 7 \operatorname{stn} 8$ (Figure 7F).

To assess the performance of PSI in oeSTN8, the photochemical quantum yield of PSI $\left(\Phi_{\mathrm{I}}\right)$ and the quantum yield of non-photochemical energy dissipation in PSI due to donor $\left(\Phi_{\mathrm{ND}}\right)$ or acceptor side limitation $\left(\Phi_{\mathrm{NA}}\right)$ were determined in WT, oeSTN8, stn8-1 and $\operatorname{stn} 7 \operatorname{stn} 8$ plants by performing a light curve with increasing light intensities (Figure S2). All PSI values determined for all mutant lines, except $\operatorname{stn} 7 \sin 8$, lay within the standard deviation of the WT. Only at weaker light intensities up to $216 \mu \mathrm{mol}$ photons $\mathrm{m}^{-2} \mathrm{~s}^{-1}$ did oeSTN8 show a slight tendency to higher (and $\operatorname{stn} 8-1$ to lower) $\Phi_{\mathrm{I}}$ values compared to WT (Figure S2).

\section{INCREASED STN8 ACTIVITY RESULTS IN HIGHER GRANA STACKS}

PSII phosphorylation, primarily mediated by STN8, has been proposed to alter the macroscopic folding of the thylakoid membrane (Fristedt et al., 2009). Specifically, a reduction in thylakoid protein phosphorylation, as in $\operatorname{stn} 8-1$, is associated with an increase in grana diameter (Fristedt et al., 2009). This effect was even more pronounced in the $\sin 7 \operatorname{stn} 8$ double mutant (Fristedt et al., 2009; Herbstova et al., 2012; Armbruster et al., 2013), which in addition has fewer membrane layers per granum and therefore shows a reduction in grana height (Armbruster et al., 2013). To determine the effects of enhanced STN8-mediated phosphorylation on thylakoid ultrastructure, WT, oeSTN8 and stn8-1 plants were adapted to LL for $1.5 \mathrm{~h}$ after $12 \mathrm{~h}$ of darkness, and the chloroplasts in thin sections of leaves were analyzed by transmission electron microscopy (Figures 8A-C). While grana stacks of WT exhibited an average ratio of diameter to height of $0.47 / 0.11$ $( \pm 0.01 / 0.01) \mu \mathrm{m}$, the corresponding value for $\operatorname{stn} 8-1$ grana was $0.58 / 0.06( \pm 0.02 / 0.00) \mu \mathrm{m}$ (Figure 8D). These observations confirmed the significant tendency toward an increase in grana diameter in $\operatorname{stn} 8$ - 1 (Fristedt et al., 2009), and the detected decrease in grana height is in good agreement with the measurements by Armbruster et al. (2013), giving a diameter/height ratio for $\operatorname{stn} 8$ - 1 that lies between those for WT and $\sin 7 \sin 8$ double mutant plants. oeSTN8 on the other hand showed an average grana diameter/height ratio of $0.54 / 0.15( \pm 0.01 / 0.01) \mu \mathrm{m}$ (Figure 8D). The moderate but significant increase in grana height compared to WT is in accordance with that seen in other mutant lines (i.e., tap38) showing enhanced thylakoid phosphorylation (Armbruster et al., 2013). However, this increase in grana height does not seem to be compensated by a decrease in grana diameter. All differences in grana diameter and height between the lines are significant at $p$-values of $<0.05$. Whether the observed changes in thylakoid ultrastructure associated with increased levels of STN8 result from (i) enhanced PSII phosphorylation, (ii) the concomitant alterations in PSII supercomplex composition or (iii) possible changes in the phosphorylation state of the CURT1 proteins, which were recently shown to mediate grana formation by inducing membrane curvature (Armbruster et al., 2013), remains to be elucidated.

\section{DISCUSSION}

\section{STN KINASES: SIMILAR BIOCHEMICAL FEATURES BUT NO FUNCTIONAL INTERDEPENDENCE}

When STL1, the STN8 homolog in Chlamydomonas reinhardtii, was shown to be phosphorylated in an Stt7-dependent manner (Lemeille et al., 2010), the idea was proposed that STN7 and STN8 might also form part of a kinase cascade (Lemeille and Rochaix, 2010). It was later shown that the phosphorylation status of STN7 is not affected in the stn8-1 mutant, indicating that STN7 is not phosphorylated by STN8 (Reiland et al., 

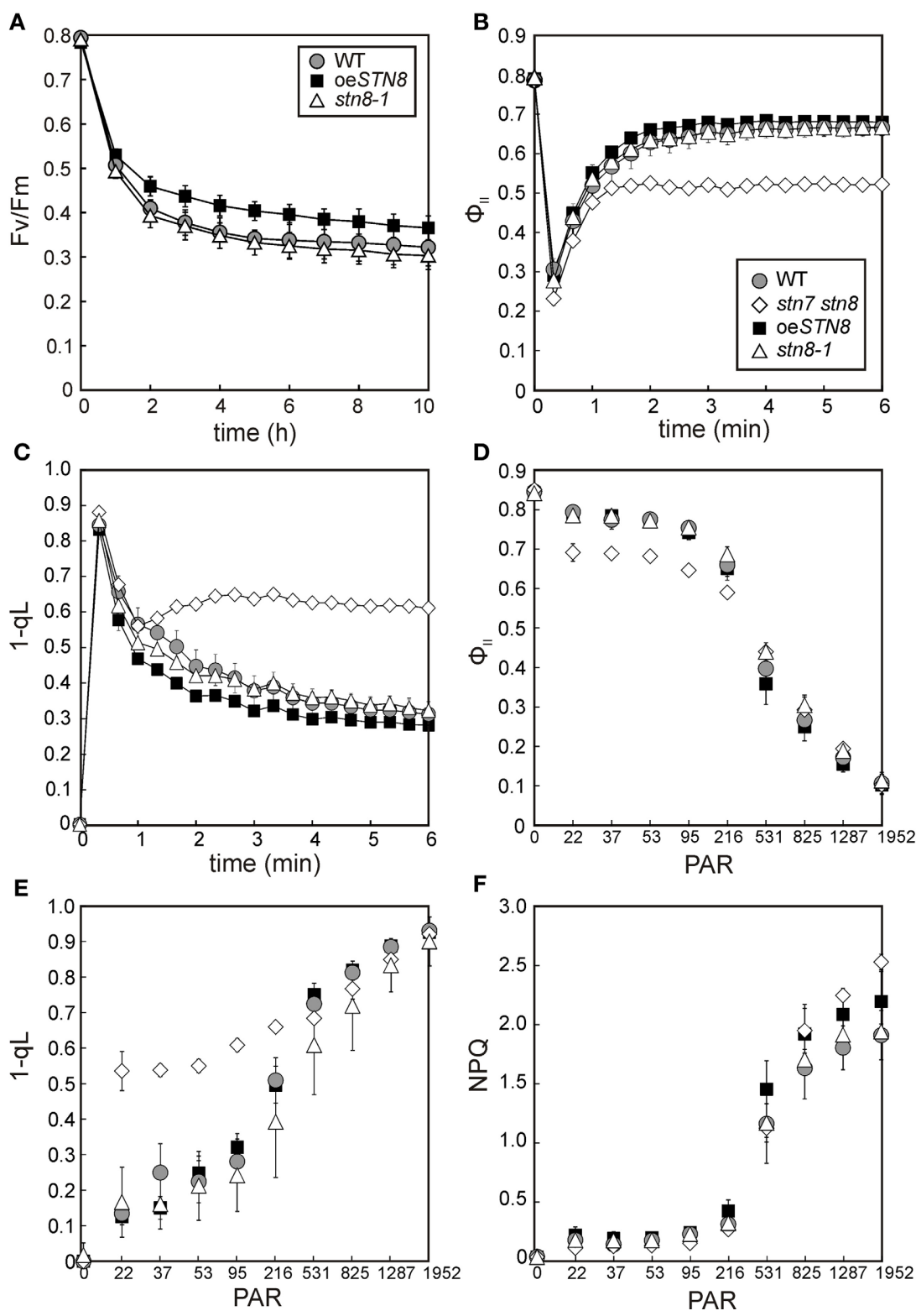

FIGURE 7 | Kinetics of $\mathrm{Chl}$ a fluorescence and time-course of PSII photoinhibition in WT, stn8-1 and oeSTN8 plants. (A) WT, stn8-1 and oeSTN8 plants were exposed to fluctuating (blue) light intensities for $10 \mathrm{~h}$ by switching from $\mathrm{HL}\left(1,250 \mu \mathrm{mol}\right.$ photons $\left.\mathrm{m}^{-2} \mathrm{~s}^{-1}\right)$ to $\mathrm{LL}(10 \mu \mathrm{mol}$ photons $\mathrm{m}^{-2} \mathrm{~s}^{-1}$ ) and back every $3 \mathrm{~min}$. The maximum quantum yield of PSII (Fv/Fm) was measured every $60 \mathrm{~min}$ after the LL phase and additional 5 min dark adaptation. $\mathrm{HL}$ exposure and PAM measurements were performed using an Imaging PAM system (Heinz Walz $\mathrm{GmbH}$ ). (B,C) Time-course of the effective quantum yield of PSII $\left(\Phi_{\|}\right)$(B) and excitation pressure of PSII (1-qL) (C) of plants pre-incubated for $10 \mathrm{~min}$ in the dark and exposed to actinic red light $\left(22 \mu \mathrm{mol}\right.$ photons $\left.\mathrm{m}^{-2} \mathrm{~s}^{-1}\right)$ for $6 \mathrm{~min}$. (D-F)

Dependence of $\mathrm{Chl}$ a fluorescence on light intensity. The effective quantum yield of PSII $\left(\Phi_{\|}\right)(D)$, the excitation pressure of PSII (1-qL) (E) and non-photochemical quenching of chlorophyll fluorescence (NPQ) (F) were monitored as red light intensity was increased stepwise at 5-min intervals $(22,37,53,95,216,513,825,1,287$, and 1,952 $\mu$ mol photons $\mathrm{m}^{-2} \mathrm{~s}^{-1}$ ) after $10 \mathrm{~min}$ of dark adaptation. PAR, photosynthetically active radiation ( $\mu \mathrm{mol}$ photons $\mathrm{m}^{-2} \mathrm{~s}^{-1}$ ); circles with gray filling, $\mathrm{WT}$; squares with black filling, oeSTN8; triangles; stn8-1; diamonds, $\operatorname{stn} 7 \operatorname{stn} 8$. (A-F) Plants were grown under an $8 \mathrm{~h} / 16 \mathrm{~h}$ day/night regime at $100 \mu \mathrm{mol}$ photons $\mathrm{m}^{-2} \mathrm{~s}^{-1}$ prior to measurements. Average values $( \pm \mathrm{SD})$ of at least five individual plants are shown.

2011). Therefore, it remains unclear whether STN7 and STN8 interact functionally, either directly or indirectly. It is however accepted that there is some substrate overlap between the two (Bonardi et al., 2005; Tikkanen et al., 2008, 2010), and this also applies to the corresponding protein phosphatases, with
TAP38/PPH1 potentially targeting STN8 substrates (Vainonen et al., 2008; Pribil et al., 2010) and overexpression of PBCP - the PSII core protein phosphatase - affecting state transitions (Samol et al., 2012). Here, we show that, despite this complex interplay of these two protein kinase/phosphatase couples, knockout or 


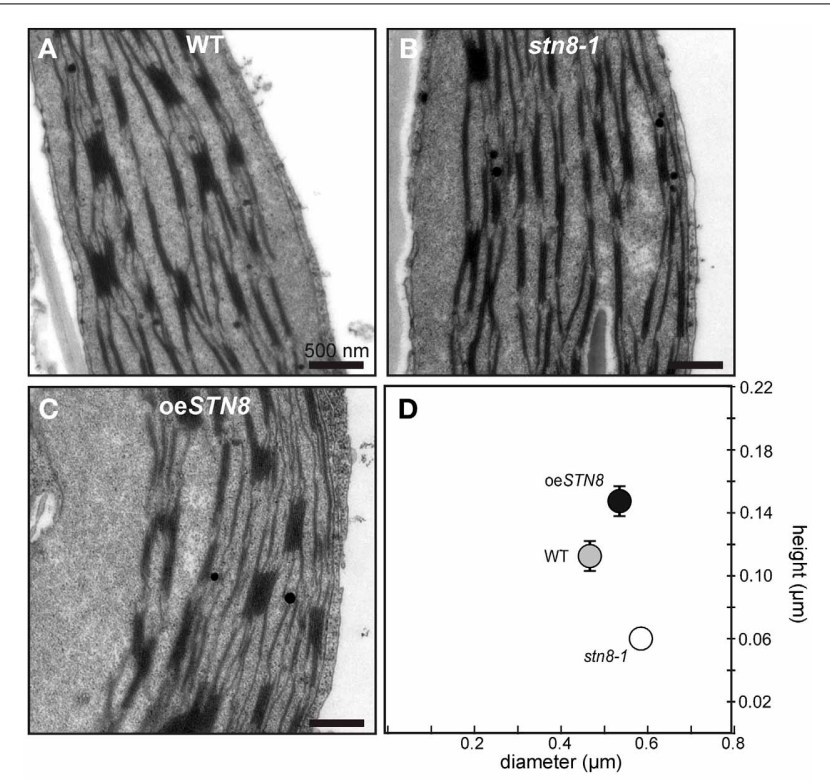

FIGURE 8 | Altered STN8 levels affect thylakoid ultrastructure. (A-C) True leaves of 4-week-old WT (Col-0), stn8-1 and oeSTN8 plants grown at $100 \mu \mathrm{mol}$ photons $\mathrm{m}^{-2} \mathrm{~s}^{-1}$ at a $12 / 12 \mathrm{~h}$ night/day cycle were fixed $2 \mathrm{~h}$ after the onset of light and analyzed by transmission electron microscopy (TEM). Representative cross-sections of chloroplasts are shown for WT (A), stn8-1 (B) and oeSTN8 (C), respectively. Bars represent $500 \mathrm{~nm}$. (D) Scatter plot of the average grana diameter and height values $(n=30)$ in the genotypes shown in (A-C). Bars represent the respective standard errors of the mean (SEM).

overexpression of one STN kinase does not significantly affect the activity or steady-state level of the other (Figure 1). Therefore, the overlap in substrate specificity of STN7 and STN8 is not reflected in a reciprocal response to changes in the level of either protein.

While the topology of Stt7 has been clarified experimentally (Lemeille et al., 2009), the assumption that STN8 is a singlepass transmembrane thylakoid protein with its kinase domain facing the stroma has been based solely on sequence predictions and the inference that PSII proteins are phosphorylated exclusively on their stromal moieties (Vainonen et al., 2005). However, the predictions of the putative transmembrane domain (TM) are not clear-cut, with some algorithms for TM prediction (i.e., TMHMM and SOSUI) suggesting that STN8 is a soluble protein. We have now confirmed that STN8 is an intrinsic thylakoid membrane protein, very similar in character to STN7 (Figure 2).

Furthermore, this study has also addressed the question whether STN7 and STN8 exist as monomeric enzymes or are associated with HMW complexes, as previously shown for Stt7 under state 1 and state 2 conditions (Lemeille et al., 2009). In 2D BN-/SDS-PAGE analyses we show that both STN kinases were associated with HMW complexes, and their presence in multiple assembly states of high molecular weight was further supported by data from sucrose-density-gradient centrifugations (Figures 3, S1). Interestingly, alterations in light quality had no significant effects on the localization of the STN kinases within the thylakoid membrane (Figure 3A). Thus, there is now comprehensive evidence that both STN7 and STN8 operate in close association with the major photosynthetic complexes. A lack of STN8 accumulation in plants devoid of PSII complexes (Figure S1) might further reflect the necessity for a close spatial relationship between STN8 and PSII core proteins, its most likely substrates. In line with these findings, STN8 was mainly detected in grana stacks or grana margins, the thylakoid fractions in which PSII accumulates (Figure 3A). These observations suggest that phosphorylation of PSII subunits by STN8 occurs directly. However, a kinase cascade residing in close proximity to the PSII complex cannot be ruled out. The latter scenario would explain results obtained by Hou et al. (2003), who showed that washing thylakoids with $2 \mathrm{M} \mathrm{NaBr}$ led to loss of the capacity for PSII core protein phosphorylation, whereas we showed here that most of the STN8 protein remained bound to the membrane after similar treatments (Figure 2B). Therefore, it is tempting to speculate that, instead of affecting the presence or activity of STN8, washing with $\mathrm{NaBr}$ removes downstream components that are part of a putative PSII core phosphorylation cascade.

\section{THE STN KINASES EXHIBIT DISTINCTLY DIFFERENT REGULATORY FEATURES}

It is generally accepted that STN7 activity can be inhibited via the stromal ferredoxin-thioredoxin pathway, but the precise site of inactivation remains a matter of speculation. Two main scenarios have been considered: (i) thioredoxins directly target the stroma-exposed cysteines Cys 187 and Cys 191, which reside within the ATP binding pocket (Puthiyaveetil, 2011) and (ii) the redox signal is transferred to the lumen via the putative CcdA/Hcf164 pathway targeting the lumenal cysteines Cys 65 and Cys 70 (Lemeille and Rochaix, 2010). Here, we have presented experimental evidence for a direct physical interaction between STN7 and recombinant thioredoxin- $\mathrm{f}$ ( $\operatorname{rec} \Delta \mathrm{TRX}-\mathrm{f})$, which is not affected by replacement of the luminal STN7 cysteines Cys 65 and Cys 70 (Figures 4B,C). This observation supports the idea that STN7 is targeted by thioredoxins at its stromal CxxxC motif, which presumably interferes with the binding of ATP to the ATP binding pocket, especially under HL conditions, and thereby leads to the inactivation of STN7. The lack of this stromal cysteine motif in Stt7 and the fact that no thioredoxin-dependent inhibition of Stt7 has been found in C. reinhardtii (Puthiyaveetil, 2011) both argue in favor of this scenario. Further evidence for disulfide bridge formation in STN7, involving cysteines other than the known N-terminally located ones, was provided by redox titration experiments, which showed that STN7 reduction results in a shift in its migration behavior even in the absence of the N-terminal cysteine motif (Figure 4A). This suggests the presence of a second redox-sensitive motif besides the confirmed $\mathrm{N}$-terminally located one, which could be regulated by thioredoxins. The observation that the electrophoretic mobility of STN7 is increased under reducing conditions is rather unusual for the opening of a disulfide bridge and might therefore be attributable to the release of an as yet unknown STN7-bound cofactor. 
At least one of two conserved cysteine motifs, both of which are absent in STN8, is thought to be involved in thioredoxinmediated down-regulation of Stt7/STN7 activity and protein levels under HL conditions (Rintamäki et al., 2000; Lemeille et al., 2009; Puthiyaveetil, 2011; Wunder et al., 2013). Thus, in contrast to STN7, STN8 activity is retained or even increased under these lighting conditions (Bonardi et al., 2005; Tikkanen et al., 2008).

As STN8 has none of the cysteine motifs that are commonly subject to redox-dependent regulation (Depège et al., 2003), it seemed possible that STN8 activity might be controlled on the level of protein amounts. However, in contrast to STN7, levels of the STN8 protein remained surprisingly stable both under activity-promoting and inactivating light conditions (Figure 5), suggesting that an activity-modulating mechanism must act on STN8. As such, reversible phosphorylation of STN8 could represent an essential mode of regulation. The phosphorylation of STL1, the STN8 homolog in C. reinhardtii, in a Stt7dependent manner (Lemeille et al., 2010) argues in favor of this scenario.

\section{PHYSIOLOGICAL EFFECTS OF VARYING STN8 PROTEIN LEVELS}

Elevated STN8 levels resulted in an overall increase in CP43, D1 and D2 phosphorylation under all light conditions tested (Figure 6A). Only FR conditions, which cause a strong oxidation of the PQ pool, led to a substantial decrease in PSII phosphorylation even in the presence of increased amounts of STN8, supporting the notion that redox-responsive mechanisms act (presumably indirectly; see above) on STN8 activity. This PSI lightdependent dephosphorylation of PSII core proteins was proposed to be relevant for the formation of PSII supercomplexes, the photosynthetically most efficient conformation of PSII (Tikkanen et al., 2008; Tikkanen and Aro, 2012). In contrast, under HL conditions, LHCII is preferentially released from the photosystems to participate in heat dissipation (Allakhverdiev and Murata, 2004; Murata et al., 2007; Allakhverdiev et al., 2008) and amounts of PSII supercomplexes are decreased due to increased PSII protein phosphorylation (Tikkanen et al., 2008, 2010). While studies on the role of PSII core phosphorylation in D1 turnover based on the protein kinase mutants $\sin 8$ and $\sin 7 \sin 8$ have led to somewhat contradictory results (Bonardi et al., 2005; Tikkanen et al., 2008; Fristedt et al., 2009), more recently the consensus has emerged that PSII phosphorylation exerts a rather indirect influence on PSII turnover via the modulation of thylakoid ultrastructure and PSII complex formation and migration (Grouneva et al., 2013). Here we show that the increased PSII core phosphorylation in oeSTN8 plants (Figure 6A) indeed leads to a slight reduction in photoinhibition after long-term exposure to fluctuating $\mathrm{HL}$ (Figure 7A). The underlying increase in D1 turnover efficiency can be ascribed to mechanisms that involve (i) PSII supercomplex disassembly and/or (ii) the modulation of thylakoid membrane stacking.

(i) While Tikkanen et al. (2008) interpreted the observed delay in D1 degradation in the $\operatorname{stn} 8-1$ mutants under HL as a consequence of slower disassembly of PSII supercomplexes, no such changes in the ratio of PSII supercomplexes to PSII monomers could be detected by Fristedt et al. (2009) after $3 \mathrm{~h}$ of HL treatment. Interestingly, the direct comparison of PSII supercomplex formation in WT, oeSTN8 and stn8-1 of this study revealed an obvious discrepancy between the respective genotypes under $\mathrm{D}$ and LL, and even more so under HL conditions (Figures 6B,C). While the disassembly of PSII supercomplexes was slightly promoted in oeSTN8, PSII supercomplexes clearly accumulated in stn8-1, suggesting clear effects of altered STN8 levels. However, these observations do not fully explain the altered resistance to photoinhibition seen for oeSTN8 but not for $\operatorname{stn} 8-1$ (Figure 7A), as both genotypes exhibit aberrant PSII supercomplex formation compared to WT.

(ii) The slightly higher resistance of oeSTN8 to photoinhibition could also be due to an increase in charge-dependent repulsion of the thylakoid membranes, which is reported to cause looser grana stacking and therefore allows faster lateral movement of proteins (Fristedt et al., 2009). In fact, compared to WT, slight changes in macroscopic thylakoid membrane folding could be observed in oeSTN8 under low light intensities (Figure 8), where differences in PSII phosphorylation between oeSTN8 and WT are only marginal (Figure 6A). Surprisingly, both height and diameter of the grana stacks seemed to be slightly increased in oeSTN8 (Figure 8), which argues against facilitated movement of membrane proteins between grana and stroma thylakoids, at least from the perspective of the grana diameter (Fristedt et al., 2009). The idea that the association of HL-induced PSII phosphorylation with altered grana stacking is purely coincidental, and that other HL-induced, STN8-independent processes are actually responsible for the modulation of grana stacking can probably be ruled out also, as oeSTN8 shows somewhat increased grana stacking already under LL (Figure 8). This provides evidence that thylakoid protein phosphorylation mediated by STN8 is indeed responsible for the observed changes in thylakoid remodeling. Nevertheless, it remains unclear whether STN8-dependent changes in grana formation actually result from alterations in PSII core protein phosphorylation or possible changes in the phosphorylation state of the CURT1 proteins, which were shown to mediate grana formation by inducing membrane curvature (Armbruster et al., 2013), or other so far unknown thylakoid components. In this respect, whether the differences in PSII supercomplex composition are the cause or the consequence of the altered macroscopic thylakoid membrane folding remains to be elucidated. In conclusion, it can be stated that the aberrant phosphorylation of PSII core proteins in oeSTN8 and stn8-1 plants has only a minor impact on photosynthetic performance (Figures 7, S2).

\section{AUTHOR CONTRIBUTIONS}

Research was designed by Tobias Wunder, Mathias Pribil and Dario Leister. Research was performed by Tobias Wunder, Wenteng Xu, Qiuping Liu, Gerhard Wanner, and Mathias Pribil. The manuscript was prepared by Mathias Pribil, Tobias Wunder, and Dario Leister. The whole study was supervised by Dario Leister.

\section{ACKNOWLEDGMENTS}

We thank the Deutsche Forschungsgemeinschaft (grant LE1265/21-1) for support and Paul Hardy for critical reading 
of the manuscript. Further we thank Prof. Dr. Roberto Barbato (Alessandria, Italy) and Dr. Paolo Pesaresi (Milan, Italy) for providing antibodies against mature STN7 and STN8 and Dr. Alexander Hertle for providing recombinant TRX-f and $-\mathrm{m}$.

\section{REFERENCES}

Adam, Z., Rudella, A., and Van Wijk, K. J. (2006). Recent advances in the study of Clp, FtsH and other proteases located in chloroplasts. Curr. Opin. Plant Biol. 9, 234-240. doi: 10.1016/j.pbi.2006.03.010

Allakhverdiev, S. I., and Murata, N. (2004). Environmental stress inhibits the synthesis de novo of proteins involved in the photodamage-repair cycle of Photosystem II in Synechocystis sp. PCC 6803. Biochim. Biophys. Acta 1657, 23-32. doi: 10.1016/j.bbabio.2004.03.003

Allakhverdiev, S. I., Kreslavski, V. D., Klimov, V. V., Los, D. A., Carpentier, R., and Mohanty, P. (2008). Heat stress: an overview of molecular responses in photosynthesis. Photosynth. Res. 98, 541-550. doi: 10.1007/s11120-008-9331-0

Armbruster, U., Labs, M., Pribil, M., Viola, S., Xu, W., Scharfenberg, M., et al. (2013). Arabidopsis CURVATURE THYLAKOID1 Proteins modify thylakoid architecture by inducing membrane curvature. Plant Cell 25, 2661-2678. doi: $10.1105 /$ tpc. 113.113118

Aronsson, H., and Jarvis, P. (2002). A simple method for isolating import-competent Arabidopsis chloroplasts. FEBS Lett. 529, 215-220. doi: 10.1016/S0014-5793 (02)03342-2

Baena-Gonzalez, E., Barbato, R., and Aro, E. M. (1999). Role of phosphorylation in the repair cycle and oligomeric structure of photosystem II. Planta 208, 196-204. doi: $10.1007 / \mathrm{s} 004250050550$

Bassi, R., Marquardt, J., and Lavergne, J. (1995). Biochemical and functional properties of photosystem II in agranal membranes from maize mesophyll and bundle sheath chloroplasts. Eur. J. Biochem. 233, 709-719. doi: 10.1111/j.1432-1033.1995.709_3.x

Bonardi, V., Pesaresi, P., Becker, T., Schleiff, E., Wagner, R., Pfannschmidt, T., et al. (2005). Photosystem II core phosphorylation and photosynthetic acclimation require two different protein kinases. Nature 437, 1179-1182. doi: 10.1038/nature04016

Clough, S. J., and Bent, A. F. (1998). Floral dip: a simplified method for Agrobacterium-mediated transformation of Arabidopsis thaliana. Plant J. 16, 735-743. doi: 10.1046/j.1365-313x.1998.00343.x

DalCorso, G., Pesaresi, P., Masiero, S., Aseeva, E., Schünemann, D., Finazzi, G., et al. (2008). A complex containing PGRL1 and PGR5 is involved in the switch between linear and cyclic electron flow in Arabidopsis. Cell 132, 273-285. doi: 10.1016/j.cell.2007.12.028

Depège, N., Bellafiore, S., and Rochaix, J. D. (2003). Role of chloroplast protein kinase Stt7 in transition in Chlamydomonas. Science 299, 1572-1575. doi: $10.1126 /$ science. 1081397

Fristedt, R., Willig, A., Granath, P., Crevecoeur, M., Rochaix, J. D., and Vener, A. V. (2009). Phosphorylation of photosystem II controls functional macroscopic folding of photosynthetic membranes in Arabidopsis. Plant Cell 21, 3950-3964. doi: 10.1105/tpc.109.069435

Grouneva, I., Gollan, P. J., Kangasjärvi, S., Suorsa, M., Tikkanen, M., and Aro, E. M. (2013). Phylogenetic viewpoints on regulation of light harvesting and electron transport in eukaryotic photosynthetic organisms. Planta 237, 399-412. doi: 10.1007/s00425-012-1744-5

Haldrup, A., Naver, H., and Scheller, H. V. (1999). The interaction between plastocyanin and photosystem I is inefficient in transgenic Arabidopsis plants lacking the PSI-N subunit of photosystem I. Plant J. 17, 689-698. doi: 10.1046/j.1365-313X.1999.00419.x

Heinemeyer, J., Eubel, H., Wehmhöner, D., Jänsch, L., and Braun, H. P. (2004). Proteomic approach to characterize the supramolecular organization of photosystems in higher plants. Phytochemistry 65, 1683-1692. doi: 10.1016/j.phytochem.2004.04.022

Herbstova, M., Tietz, S., Kinzel, C., Turkina, M. V., and Kirchhoff, $\mathrm{H}$. (2012). Architectural switch in plant photosynthetic membranes induced Sci. U.S.A. 109, 20130-20135. doi: 10.1073/pnas.1214265109 E. M. (2003). Ascorbate-mediated LHCII protein phosphorylationLHCII kinase regulation in light LHCII phosphorylation and state by light stress. Proc. Natl. Acad.

Hou, C. X., Rintamäki, E., and Aro,

\section{SUPPLEMENTARY MATERIAL}

The Supplementary Material for this article can be found online at: http://www.frontiersin.org/journal/10.3389/ fpls.2013.00417/abstract

and in darkness. Biochemistry 42, 5828-5836. doi: 10.1021/bi0343119 Ihnatowicz, A., Pesaresi, P., Lohrig, K., Wolters, D., Müller, B., and Leister, D. (2008). Impaired photosystem I oxidation induces STN7-dependent phosphorylation of the light-harvesting complex I protein Lhca4 in Arabidopsis thaliana. Planta 227, 717-722. doi: 10.1007/s00425-007-0650-8

Ihnatowicz, A., Pesaresi, P., Varotto, C., Richly, E., Schneider, A., Jahns, P., et al. (2004). Mutants for photosystem I subunit D of Arabidopsis thaliana: effects on photosynthesis, photosystem I stability and expression of nuclear genes for chloroplast functions. Plant J. 37, 839-852. doi: 10.1111/j.1365-313X.2004.02011.x

Karnauchov, I., Herrmann, R. G., and Klösgen, R. B. (1997). Transmembrane topology of the Rieske $\mathrm{Fe} / \mathrm{S}$ protein of the cytochrome b6/f complex from spinach chloroplasts. FEBS Lett. 408, 206-210. doi: 10.1016/S0014-5793(97)00427-4

Koivuniemi, A., Aro, E. M. and Andersson, B. (1995). Degradation of the D1- and D2-proteins of photosystem II in higher plants is regulated by reversible phosphorylation. Biochemistry 34, 16022-16029. doi: 10.1021/bi00049a016

Laemmli, U. K. (1970). Cleavage of structural proteins during the assembly of the head of bacteriophage T4. Nature 227, 680-685. doi: 10.1038/227680a0

Lemeille, S., and Rochaix, J. D. (2010) State transitions at the crossroad of thylakoid signalling pathways. Photosynth. Res. 106, 33-46. doi: 10.1007/s11120-010-9538-8

Lemeille, S., Turkina, M. V., Vener, A. V., and Rochaix, J. D. (2010). Stt7dependent phosphorylation during state transitions in the green alga Chlamydomonas reinhardtii. Mol. Cell Proteomics 9, 1281-1295. doi: 10.1074/mcp.M000020-MCP201

Lemeille, S., Willig, A., DepègeFargeix, N., Delessert, C., Bassi, R., and Rochaix, J. D. (2009). Analysis of the chloroplast protein kinase Stt7 during state transitions. PLoS Biol. 7:e45. doi: 10.1371/journal.pbio.1000045

Maiwald, D., Dietzmann, A., Jahns, P., Pesaresi, P., Joliot, P., Joliot, A., et al. (2003). Knock-out of the genes coding for the Rieske protein and the ATP-synthase delta-subunit of Arabidopsis. effects on photosynthesis, thylakoid protein composition, and nuclear chloroplast gene expression. Plant Physiol. 133, 191-202. doi: 10.1104/pp.103.024190

Meurer, J., Plücken, H., Kowallik, K. V., and Westhoff, P. (1998). A nuclearencoded protein of prokaryotic origin is essential for the stability of photosystem II in Arabidopsis thaliana. EMBO J. 17, 5286-5297. doi: 10.1093/emboj/17.18.5286

Motohashi, K., Kondoh, A., Stumpp, M. T., and Hisabori, T. (2001). Comprehensive survey of proteins targeted by chloroplast thioredoxin. Proc. Natl. Acad. Sci. U.S.A. 98, 11224-11229. doi: 10.1073/pnas. 191282098

Murata, N., Takahashi, S., Nishiyama, Y., and Allakhverdiev, S. I. (2007). Photoinhibition of photosystem II under environmental stress. Biochim. Biophys. Acta 1767, 414-421. doi: 10.1016/j.bbabio.2006.11.019

Nath, K., Mishra, S., Zulfugarov, I., Raffi, S., An, G., and Lee, C. H. (2007). Characterization of a TDNA inserted STN8 kinase mutant of Ozyra sativa L. Photosynth. Res. 91, 289.

Nixon, P. J., Barker, M., Boehm, M., De Vries, R., and Komenda, J. (2005). FtsH-mediated repair of the photosystem II complex in response to light stress. J. Exp. Bot. 56, 357-363. doi: 10.1093/jxb/ eri021

Porra, R. J. (2002). The chequered history of the development and use of simultaneous equations for the accurate determination of chlorophylls $\mathrm{a}$ and $\mathrm{b}$. Photosynth. Res. 73, 149-156. doi: 10.1023/A:1020470224740

Pribil, M., Pesaresi, P., Hertle, A., Barbato, R., and Leister, D. (2010). Role of plastid protein phosphatase TAP38 in LHCII dephosphorylation and thylakoid electron flow. PLoS Biol. 8:e1000288. doi: 10.1371/journal.pbio. 1000288

Puthiyaveetil, S. (2011). A mechanism for regulation of chloroplast LHC II kinase by plastoquinol and thioredoxin. FEBS Lett. 585, 1717-1721. doi: 10.1016/j.febslet.2011.04.076 
Reiland, S., Finazzi, G., Endler, A., Willig, A., Baerenfaller, K., Grossmann, J., et al. (2011). Comparative phosphoproteome profiling reveals a function of the STN8 kinase in fine-tuning of cyclic electron flow (CEF). Proc. Natl. Acad. Sci. U.S.A. 108, 12955-12960. doi: 10.1073/pnas.1104734108

Rintamäki, E., Kettunen, R., and Aro, E. M. (1996). Differential D1 dephosphorylation in functional and photodamaged photosystem II centers. Dephosphorylation is a prerequisite for degradation of damaged D1. J. Biol. Chem. 271, 14870-14875. doi: 10.1074/jbc.271.25.14870

Rintamäki, E., Martinsuo, P., Pursiheimo, S., and Aro, E. M. (2000). Cooperative regulation of light-harvesting complex II phosphorylation via the plastoquinol and ferredoxinthioredoxin system in chloroplasts. Proc. Natl. Acad. Sci. U.S.A. 97, 11644-11649. doi: 10.1073/pnas. 180054297

Samol, I., Shapiguzov, A., Ingelsson, B., Fucile, G., Crevecoeur, M., Vener, A. V., et al. (2012). Identification of a photosystem II phosphatase involved in light acclimation in Arabidopsis. Plant Cell 24, 2596-2609. doi: 10.1105/tpc.112.095703

Schaffner, W., and Weissmann, C. (1973). A rapid, sensitive, and specific method for the determination of protein in dilute solution.
Anal. Biochem. 56, 502-514. doi: 10.1016/0003-2697(73)90217-0

Schottkowski, M., Gkalympoudis, S., Tzekova, N., Stelljes, C., Schünemann, D., Ankele, E., et al. (2009a). Interaction of the periplasmic PratA factor and the PsbA (D1) protein during biogenesis of photosystem II in Synechocystis sp. PCC 6803. J. Biol. Chem. 284, 1813-1819. doi: 10.1074/jbc.M806116200

Schottkowski, M., Ratke, J., Oster, U., Nowaczyk, M., and Nickelsen, J. (2009b). Pitt, a novel tetratricopeptide repeat protein involved in lightdependent chlorophyll biosynthesis and thylakoid membrane biogenesis in Synechocystis sp. PCC 6803. Mol. Plant 2, 1289-1297. doi: $10.1093 / \mathrm{mp} / \mathrm{ssp} 075$

Shapiguzov, A., Ingelsson, B., Samol, I., Andres, C., Kessler, F., Rochaix, J. D., et al. (2010). The PPH1 phosphatase is specifically involved in LHCII dephosphorylation and state transitions in Arabidopsis. Proc. Natl. Acad. Sci. U.S.A. 107, 4782-4787. doi: 10.1073/pnas. 0913810107

Tikkanen, M., and Aro, E. M. (2012). Thylakoid protein phosphorylation in dynamic regulation of photosystem II in higher plants. Biochim. Biophys. Acta 1817, 232-238. doi: 10.1016/j.bbabio.2011.05.005

Tikkanen, M., Grieco, M., Kangasjärvi, S., and Aro, E. M. (2010). Thylakoid protein phosphorylation in higher plant chloroplasts optimizes electron transfer under fluctuating light. Plant Physiol. 152, 723-735. doi: 10.1104/pp.109.150250

Tikkanen, M., Nurmi, M., Kangasjärvi, S., and Aro, E. M. (2008). Core protein phosphorylation facilitates the repair of photodamaged photosystem II at high light. Biochim. Biophys. Acta 1777, 1432-1437. doi: 10.1016/j.bbabio.2008.08.004

Vainonen, J. P., Hansson, M., and Vener, A. V. (2005). STN8 protein kinase in Arabidopsis thaliana is specific in phosphorylation of photosystem II core proteins. J. Biol. Chem. 280, 33679-33686. doi: 10.1074/jbc.M505729200

Vainonen, J. P., Sakuragi, Y., Stael, S., Tikkanen, M., Allahverdiyeva, Y., Paakkarinen, V., et al. (2008). Light regulation of $\mathrm{CaS}$, a novel phosphoprotein in the thylakoid membrane of Arabidopsis thaliana. FEBS J. 275, 1767-1777. doi: 10.1111/j.1742-4658.2008.06335.x

Wagner, R., Dietzel, L., Bräutigam, K., Fischer, W., and Pfannschmidt, T. (2008). The long-term response to fluctuating light quality is an important and distinct light acclimation mechanism that supports survival of Arabidopsis thaliana under low light conditions. Planta 228 573-587. doi: 10.1007/s00425-0080760-y

Wunder, T., Liu, Q., Aseeva, E., Bonardi, V., Leister, D., and Pribil, M. (2013). Control of STN7 transcript abundance and transient STN7 dimerisation are involved in the regulation of STN7 activity. Planta 237, 541-558. doi: 10.1007/s00425-012-1775-y

Conflict of Interest Statement: The authors declare that the research was conducted in the absence of any commercial or financial relationships that could be construed as a potential conflict of interest.

Received: 16 August 2013; accepted: 01 October 2013; published online: 21 October 2013.

Citation: Wunder T, Xu W, Liu Q, Wanner G, Leister D and Pribil M (2013) The major thylakoid protein kinases STN7 and STN8 revisited: effects of altered STN8 levels and regulatory specificities of the STN kinases. Front. Plant Sci. 4:417. doi: 10.3389/fpls.2013.00417 This article was submitted to Plant Physiology, a section of the journal Frontiers in Plant Science.

Copyright (c) 2013 Wunder, Xu, Liu, Wanner, Leister and Pribil. This is an open-access article distributed under the terms of the Creative Commons Attribution License (CC BY). The use, distribution or reproduction in other forums is permitted, provided the original author(s) or licensor are credited and that the original publication in this journal is cited, in accordance with accepted academic practice. No use, distribution or reproduction is permitted which does not comply with these terms. 